\begin{tabular}{|c|c|c|c|c|c|}
\hline MUNIBE Antropologia-Arkeologia & $n^{\circ} 66$ & 309-325 & DONOSTIA & 2015 & ISSN 1132-2217 • eISSN 2172-4555 \\
\hline
\end{tabular}

\title{
Producción y uso de ornamentos en las tierras bajas de Sudamérica: el caso de las poblaciones humanas prehispáni- cas del extremo meridional de la cuenca del Plata (Argentina)
}

\author{
The production and use of ornaments in the lowlands of South America: the case of \\ pre-Hispanic human populations of the southern end of the La Plata basin (Argentina)
}

PALABRAS CLAVES: Ornamento, cazadores-recolectores, tierras bajas, señalización costosa.

GAKO-HITZAK: apaingarria, ehiztariak-biltzaileak, beheko lurrak, seinaleztapen neketsua.

KEY WORDS: Ornaments, hunter-gatherer, lowlands, costly signaling.

Alejandro A. ACOSTA(1), Natacha BUC(1) \& M. Natalia DAVRIEUX(1)

\section{RESUMEN}

En este trabajo se analizan los conjuntos ornamentales generados por las poblaciones humanas prehispánicas que ocuparon el extremo meridional de la cuenca del Plata. Se describen las piezas recuperadas en diversos sitios arqueológicos teniendo en cuenta las materias primas empleadas, sus mecanismos de obtención y otros aspectos relacionados con la manufactura. Posteriormente se evalúa y discute su potencial uso como medios de transmisión de información. Para ello se consideran diferentes factores vinculados con los sistemas de intercambio y la extensión de las redes sociales en el marco del crecimiento demográfico y del grado de complejidad social que alcanzaron dichas poblaciones durante la fase final del Holoceno tardío.

\section{LABURPENA}

Lan honetan Cuenca del Platako hegoaldeko muturrean bizi izan ziren giza populazio prehispanikoek sortutako multzo apaingarriak aztertzen dira. Toki arkeologiko ugaritan berreskuratutako piezak deskribatzen dira erabilitako lehengaiak, lortzeko mekanismoak eta manufakturarekin lotutako beste alderdi batzuk kontuan izanda. Ondoren, informazioa transmititzeko baliabide gisa izan dezaketen erabilera ebaluatzen eta eztabaidatzen da. Horretarako, truke-sistemekin eta sare sozialen hedapenarekin lotutako hainbat faktore hartu da kontuan hazkunde demografikoaren eta Holozeno berantiarraren amaierako fasean populazio horiek lortu zuten konplexutasun sozial mailaren testuinguruaren baitan.

\section{ABSTRACT}

This paper analyses ornamental assemblages from human prehispanic groups that inhabited the southern portion of Del Plata basin. Artifacts recovered in different archaeological sites are described, focusing on the raw materials used, their acquisition, and other aspects linked to the manufacturing processes. Then we test and discuss their possible use as vector of information transmission. We consider different factors associated with exchange systems and the scope of social networks in the frame of a context of demographic growth and social complexity at which arrived social groups under study during the last portion of Late Holocene.

\section{1.- INTRODUCCIÓN}

Los objetos ornamentales o adornos personales constituyen una de las primeras evidencias relacionadas con la emergencia del comportamiento simbólico entre los seres humanos modernos (D'ERRICO et al., 2003; HENSHILWOOD et al., 2004; ROSSANO, 2010; VANHAEREN et al., 2013; entre otros). En estudios recientes, dichos elementos han sido considerados parte de la tecnología de señalización costosa (QUINN 2006, KUHN 2014). Diferentes autores sostienen que los adornos constituyeron un medio eficaz para transmitir información, inter e intragrupal, relacionada con distintos aspectos conductuales como, por ejemplo, identidad étnica, jerarquía, rol o prestigio social de los individuos, independientemente del significado que debieron tener para sus portadores (STINER et al., 2013; KUHN, 2014; STINER, 2014). El objetivo de este trabajo es abordar parte de las problemáticas mencionadas mediante el análisis de los conjuntos ornamentales generados por las poblaciones humanas que ocuparon un pequeño sector del extremo meridional de la cuenca del Plata en los últimos 2500 años C14AP aproximadamente.

La cuenca del Plata constituye la segunda cuenca hidrográfica más grande de Sudamérica y del mundo. Territorialmente abarca unos $3.200 .000 \mathrm{Km}^{2}$ y comprende el centro y norte de Argentina, el sudeste de Bolivia, casi toda la región meridional de Brasil, todo Paraguay y gran parte de Uruguay (Figura 1). Se encuentra integrada por cuatro subcuencas principales, representadas por los sistemas fluvia-

(1) CONICET-Instituto Nacional de Antropología y Pensamiento Latinoamericano. acosta@retina.ar 


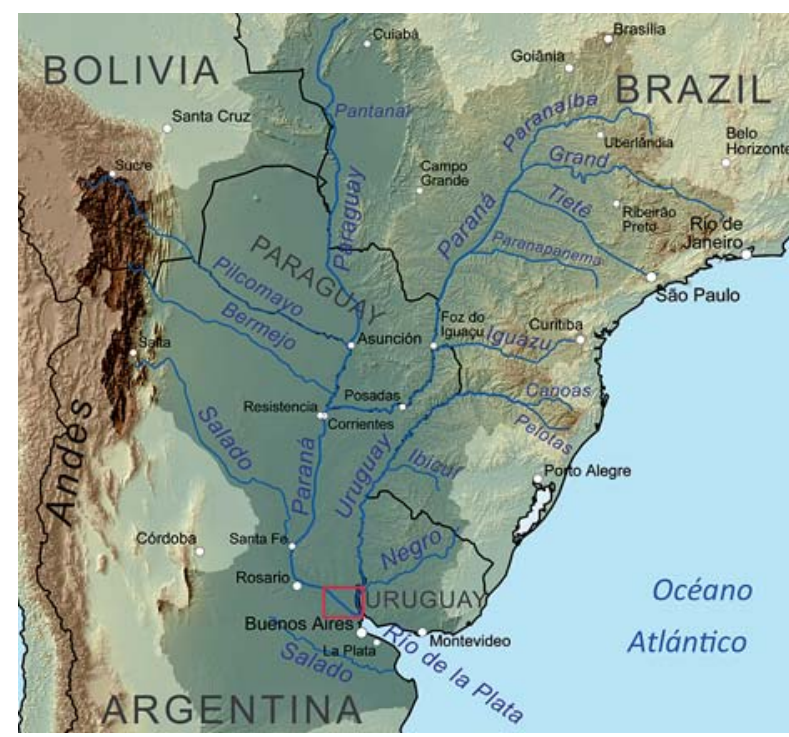

Fig. 1. Cuenca del Plata, el recuadro corresponde al humedal del Paraná inferior. Tomado y modificado de Kmusser (2010). / Del Plata basin, the Low Paraná wetland in the quadrant. Modified after Kmusser (2010).

les del Paraná, Paraguay, Uruguay y la propia subcuenca del Río de la Plata; siendo la del río Paraná la de mayor extensión, ya que cubre el 48,7\% de la superficie total de toda la cuenca. En este trabajo nos referiremos a la porción terminal de esta gran cuenca. Más específicamente, el área de estudio corresponde al tramo final del humedal del Paraná inferior (en adelante HPI) quedando comprendido, desde el punto de vista biogeográfico, dentro de la unidad ecoregional denominada "Delta e Islas del Paraná" (sensu BURKART et al., 1999). Las características geológicas y ambientales que actualmente presenta el HPI comenzaron a generarse hace unos 3500 años AP, durante la fase regresiva del nivel marino correspondiente a la ingresión marina de Holoceno temprano, cuyo inicio fue fijado en 8000 años AP. Junto con el descenso del nivel del mar, aumentaron las precipitaciones en las cabeceras del río Paraná, modificando las condiciones ambientales de estuáricas a netamente fluviales hasta alcanzar la fisonomía que hoy presenta el HPI (CAVALLOTTO et al. 1999, 2004; VIOLANTE \& PARKER, 2004). Datos paleoambientales y arqueológicos sugieren que hacia los 2500 años AP se habían desarrollado y establecido las condiciones climáticas y una biocenosis similar a la actual (TONELLO \& PRIETO, 2010, LOPONTE et al., 2012). Asimismo, diferentes líneas de evidencias arqueológicas indican que en la medida en que se fue consolidando el ambiente, los nuevos espacios que emergieron fueron rápidamente ocupados (cf. LOPONTE et al., 2012, ACOSTA \& LOPONTE, 2013). La abundante y concentrada oferta de recursos (fluviales y terrestres) que brindó el HPI jugó un papel de gran importancia en los procesos de adaptación de los grupos cazadores-recolectores que colonizaron el área. Debe tenerse en cuenta que el macrosistema de humedales generado por las redes hídricas del Paraná, se encuentra entre los ambientes más productivos del mundo (MITSCH \& GOSSELINK, 1986; NEIFF, 1999) as- pecto que, demográficamente, debió incentivar la concentración humana en el paisaje.

Dentro del escenario descripto se ha propuesto que las poblaciones humanas que ocuparon el HPI desarrollaron estrategias densodependientes basadas en un sistema de asentamientos de lugar central con una alta estabilidad residencial. A su vez, distintas evidencias sugieren un proceso de intensificación en la explotación del ambiente (ACOSTA, 2005; LOPONTE et al., 2006; LOPONTE, 2008). Este proceso se advierte, entre otros aspectos, en el progresivo aumento en el aprovechamiento sistemático de los peces (Characiformes y Siluriformes) y en el uso y procesamiento intensivo de otras presas tales como los cérvidos (Ozotoceros bezoarticus y Blastoceros dichotomus), roedores pequeños y medianos (Cavia aperea y Mycostor coypus) y moluscos fluviales (ACOSTA, 2005; LOPONTE, 2008; LOPONTE et al., 2012). Asimismo, estudios isotópicos y arqueobótanicos indican que los vegetales constituyeron un importante segmento de la dieta. Estos recursos debieron obtenerse mediante su recolección y a partir de pequeños parches productivos generados a través de prácticas agrícolas de pequeña escala, incluyendo muy posiblemente la manipulación de algunas especies silvestres (e.g. Syagrus romanzoffiana) (ZUCOL \& LOPONTE, 2005; LOPONTE \& ACOSTA, 2007; ACOSTA \& RIOS ROMAN, 2013). Estas situaciones, a su vez, implicaron de la elaboración de un equipamiento tecnológico sofisticado y eficiente caracterizado por un variado sistema de armas y por una alta producción de recipientes cerámicos que permitieron reducir el riesgo en la obtención de las presas y maximizar el retorno energético de los distintos alimentos (vegetales y animales) explotados (PÉREZ \& CAÑARDO, 2004; LOPONTE, 2008; BUC, 2012; SACUR SIVESTRE et al., 2013).

El proceso de intensificación posibilitó la conformación de grandes núcleos poblacionales con organizaciones social y económicamente complejas (LOPONTE et al., 2006; LOPONTE, 2008). Bajo estas condiciones se produjo una mayor fragmentación y diferenciación social entre los distintos grupos humanos y microsectores que comprende el $\mathrm{HPI}$, se extendieron los comportamientos cooperativos y de reciprocidad, y generaron conductas de territorialidad y conflictos por los espacios productivos. Dichas situaciones se habrían agudizado hace unos 700 años AP con el arribo al HPI de los horticultores amazónicos, históricamente conocidos como carios o guaraníes de las islas (LOPONTE et al., 2011; ACOSTA \& LOPONTE, 2013). Dentro de este contexto se intensificaron las relaciones sociales, los circuitos de intercambio de productos regionales y extra-regionales y se extendieron las redes de interacción intra e intergrupales (LOPONTE et al., 2006; LOPONTE, 2008). Dado que la utilización de objetos ornamentales constituyó parte de estas conductas, en este estudio evaluamos y discutimos diferentes aspectos relacionados con su adquisición, manufactura y uso como potenciales medios de transmisión de información. Para ello se tiene en cuenta su distribución espacial y temporal (ver Figura 2; Tabla I) y se analizan los conjuntos en sus aspectos físicos, morfológicos, estilísticos y de rastros de uso. 


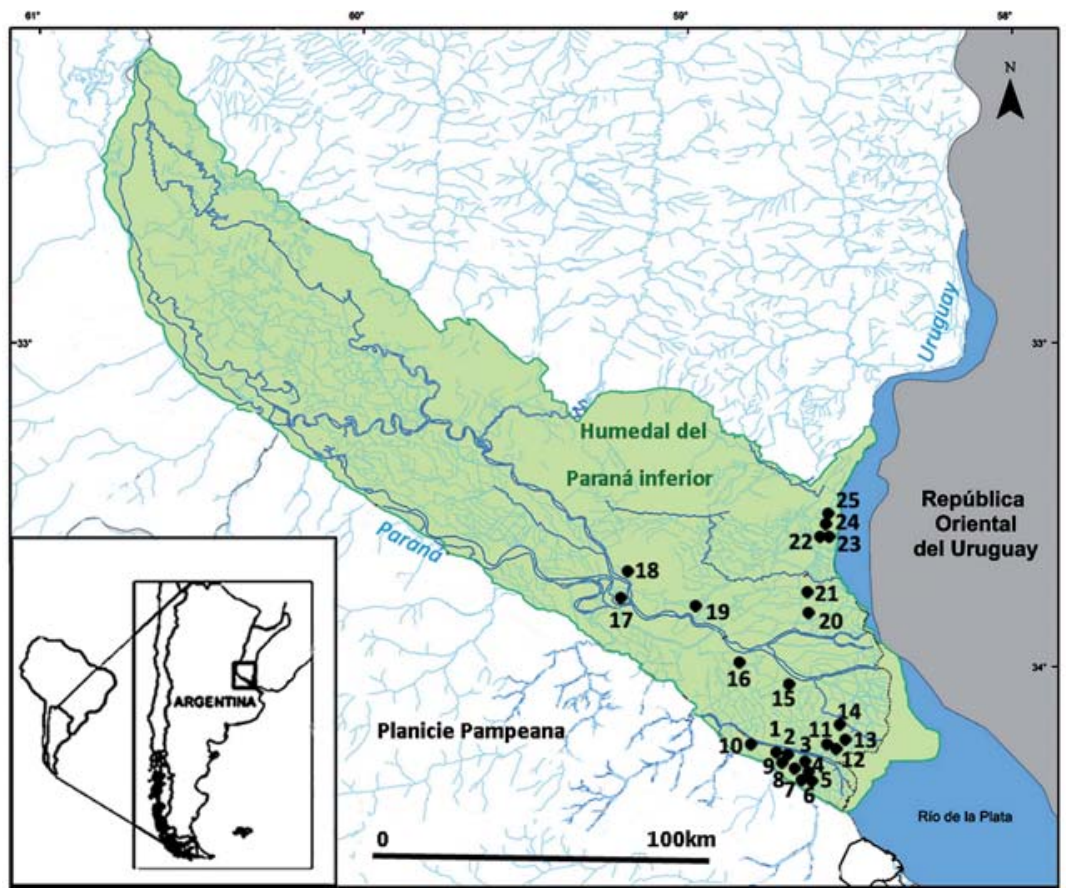

Fig. 2. Sitios arqueológicos analizados en el trabajo: 1) Anahí, 2) Las Vizcacheras, 3) Guazunambí, 4) La Bellaca I, 5) La Bellaca II, 6) Garín, 7) Punta Canal, 8 Arroyo Sarandí, 9) El Cazador 3, 10) Río Luján, 11) Arroyo Malo, 12) La Glorieta, 13) Arroyo Largo, 14) Túmulo I del Paraná Guazú, 15) Túmulo || del Paraná Guazú, 16) Cementerio del Paraná Guazú, 17) Isla Lechiguanas 1, 18) La Argentina (Paraná lbicuy 1), 19) Túmulo I del Brazo Gutiérrez, 20) Brazo Largo (Esta localización es la del sitio Túmulo I del Brazo Largo excavado por Torres (1911). Sin embargo la colección referida en este trabajo corresponde con la denominada Brazo Largo, recuperada por Gaggero y Fernández. No es claro si ambas son las mismas colecciones.), 21) Las Ánimas, 22) Cerro Lutz, 23) Cerro de los Indios, 24) Cerro Mayor. / Archaeological sites under analysis: 1) Anahí, 2) Las Vizcacheras, 3) Guazunambí, 4) La Bellaca I, 5) La Bellaca II. 6) Garín, 7) Punta Canal, 8) Arroyo Sarandí, 9) El Cazador 3, 10) Río Luján, 11 Arroyo Malo, 12) La Glorieta, 13) Arroyo Largo, 14) Túmulo | del Paraná Guazú, 15) Túmulo II del Paraná Guazú, 16) Cementerio del Paraná Guazú, 17) Isla Lechiguanas 1, 18) La Argentina (Paraná lbicuy 1), 19) Túmulo I del Brazo Gutiérrez, 20) Brazo Largo (This is the Túmulo I del Brazo site location Largo excavated by Torres (1911). However, the collection referred to in this work corresponds to the so-called Brazo Largo, recovered by Gaggero and Fernández. It is unclear whether they are both the same collections), 21) Las Ánimas, 22) Cerro Lutz, 23) Cerro de los Indios, 24) Cerro Mayor.

\begin{tabular}{|c|c|c|c|c|}
\hline SITE & C14Age & MATERIAL & LAB. & REFERENCE \\
\hline Anahi (A) & $1020 \pm 70$ & M. coypus & Beta 147108 & Loponte, 2008 \\
\hline \multirow{3}{*}{ Arroyo Fredes $\left(A^{\circ} \mathrm{F}\right)$} & $690 \pm 70$ & Homo sapiens MLP & UGA 10789 & Loponte et al, 2011 \\
\hline & $370 \pm 50$ & Homo sapiens & LP 1428 & Loponte et al, 2011 \\
\hline & $402 \pm 40$ & H. hydrochaeris & AA 77309 & Loponte et al, 2011 \\
\hline \multirow{2}{*}{ Arroyo Sarandí (A S) } & $760 \pm 37$ & M. coypus & AA 103654 & Loponte et al, 2011 \\
\hline & $1290 \pm 40$ & Homo sapiens & UGA 10788 & Loponte, 2008 \\
\hline Arroyo Largo $\left(\mathrm{A}^{\circ} \mathrm{L}\right)$ & & sin datos & & Outes, 1918 \\
\hline Brazo Largo (BL) & & sin datos & & Gaggero \& Fernández, MLR \\
\hline \multirow{4}{*}{ Cerro Lutz (CL) } & $795 \pm 42$ & Homo sapiens & AA 77311 & \multirow{4}{*}{ Acosta et al., 2011} \\
\hline & $916 \pm 42$ & Canis familiaris & AA 77312 & \\
\hline & $916 \pm 42$ & Homo sapiens & AA 77310 & \\
\hline & $1110 \pm 45$ & Homo sapiens & AA 103643 & \\
\hline Cerro de los Indios (Cl) & & sin datos & & Loponte \& Acosta, 2007 \\
\hline \multirow{5}{*}{ Cerro Mayor (CM) } & $1561 \pm 45$ & B. dichotomus & AA 97469 & \multirow{5}{*}{ Acosta et al., 2013} \\
\hline & $1574 \pm 45$ & B. dichotomus & AA 97457 & \\
\hline & $1665 \pm 45$ & Homo sapiens & AA 97468 & \\
\hline & $1600 \pm 33$ & M. coypus & AA 97466 & \\
\hline & $1594 \pm 59$ & Canis familiaris & AA 103658 & \\
\hline Cementerio del Paraná Guazú (CPG) & & sin datos & & Torres, 1911 \\
\hline \multirow{4}{*}{ El Cazador 3 (EC3) } & $1091 \pm 43$ & L. guanicoe & AA 103656 & Acosta et al., 2014 \\
\hline & $1063 \pm 46$ & L. guanicoe & AA 103657 & Acosta et al., 2014 \\
\hline & $1031 \pm 36$ & Homo sapiens & AA 97464 & Mazza \& Loponte, 2013 \\
\hline & $921 \pm 43$ & B. dichotomus & AA 97470 & Loponte \& Corriale, 2013 \\
\hline Garín (G) & $1060 \pm 60$ & M. coypus & UGA 9905 & Loponte, 2008 \\
\hline Guazunambí (Gz) & $940 \pm 60$ & B. dichotomus & Beta 147109 & Loponte, 2008 \\
\hline Isla Lechiguanas 1 (IL 1$)^{*}$ & $408 \pm 30$ & M. coypus & AA 97462 & Loponte et al., 2012 \\
\hline La Bellaca I (LBI) & $1100 \pm 70$ & Mammalia & LP 1288 & Loponte, 2008 \\
\hline La Bellaca II (LBI) & $680 \pm 80$ & B. dichotomus & LP 1263 & Loponte, 2008 \\
\hline \multirow{2}{*}{ La Argentina (AR) } & $979 \pm 44$ & Homo sapiens & AA 103642 & Mazza \& Loponte, 2013 \\
\hline & $1645 \pm 34$ & B. dichotomus & AA 97463 & Loponte \& Corriale, 2013 \\
\hline La Glorieta (LG) & $416 \pm 41$ & H. sapiens & AA 93216 & Bonomo 2013 \\
\hline Las Animas (LAN) & $1121 \pm 31$ & H. sapiens & AA 97460 & Mazza \& Loponte, 2013 \\
\hline \multirow{2}{*}{ Las Vizcacheras (LV) } & $1070 \pm 60$ & L. guanicoe & Beta 148237 & Loponte, 2008 \\
\hline & $1060 \pm 60$ & Siagrus Romanzoffiana & LP 1401 & Loponte, 2008 \\
\hline Punta Canal (PC) & $900 \pm 80$ & B. dichotomus & LP 1293 & Arrizurieta et al., 2010 \\
\hline Río Luján (RL) & $1692 \pm 46$ & B. dichotomus & AA 97458 & Loponte, 2008 \\
\hline Túmulo I Paraná Guazú (T1PG) & $576 \pm 42$ & Homo sapiens & AA 93215 & Bonomo et al., 2013 \\
\hline Túmulo I Brazo Gutiérrez (T1BG) & $752 \pm 41$ & Homo sapiens & AA 72635 & Bernal, 2008 \\
\hline Túmulo II Paraná Guazú (T2PG) & $846 \pm 41$ & Homo sapiens & AA 72633 & Bernal, 2008 \\
\hline
\end{tabular}

Tabla 1: Listado de los sitios con cronología en donde fueron recuperados los conjuntos ornmentales que aquí se estudian. *El sitio Isla Lechiguanas comprende dos niveles de ocupación arqueológica separados por 2000 años aproximadamente; los elementos ornamentales fueron recuperados sólo en el nivel superior. A list of sites where the studied ornamental sets were recovered with a time line. ${ }^{*}$ There are two levels of archaeological occupation on the Lechiguanas Islands, which are around 2000 years apart, the ornamental elements were only recovered from the upper level. 


\subsection{Aproximaciones teóricas sobre el surgimiento y uso de los adornos}

La aparición y uso de objetos simbólicos, como es el caso de los adornos, en la historia de la evolución humana en los últimos 100,000 años, representó un cambio importante en la vida social y tecnológica, constituyendo una de las respuestas ante la creciente complejidad social, económica y demográfica que experimentaron los seres humanos anatómicamente modernos (KUHN, 2014; STINER, 2014; STERELNY, 2014). Una de las hipótesis más plausibles para explicar la rápida evolución que tuvieron los sistemas simbólicos (y sus referentes materiales) es que su principal función fue la de comunicar información emblemática sobre la membrecía y el comportamiento de los individuos en la medida en que aumentó la interacción social con otros grupos humanos (cf. BETTINGER et al., 1996).

Diversos autores coinciden en que los adornos personales y otros materiales "no utilitarios" o de origen exótico fueron medios de comunicación utilizados para transmitir información de diversa índole (e.g. adscripción étnica, jerarquía o rol social de los individuos, diferenciación por género, connotaciones rituales, entre otros aspectos, TRUBBIT, 2003; WHITE, 2007; KUHN \& STINER, 2007a, 2007b). El diseño y estilo de los artefactos también han sido vinculados con la transmisión de información social (cf. WOBST, 1977). Esto último, según estudios etnográficos y arqueológicos, incluye tanto elementos "no utilitarios" como objetos funcionales, por ejemplo, puntas de proyectil (e.g. WIESSNER, 1983), bifaces (e.g. QUINN, 2015) y contenedores cerámicos (e.g. HEGMON, 1992).

Los adornos pueden fluir a lo largo de las redes sociales, ya sea como ítems por separado o en composiciones (e.g. abalorios), pudiendo constituir parte del intercambio de dones entre grupos. Este hecho, a su vez, puede implicar la continuidad de conexiones y/o de contactos duraderos y la posibilidad de que se generen o establezcan relaciones ventajosas en el futuro (KUHN, 2014), situación que permitiría reforzar los lazos sociales.

En distintos estudios se ha comenzado a explorar el potencial que brinda la teoría de la señalización costosa (en adelante TSC), desarrollada en el campo de la biología evolutiva, para explicar distintas problemáticas antropológicas (e.g. BLIEGE BIRD et al., 2001; SMITH et al., 2003; BLIEGE BIRD \& SMITH, 2005). La TSC en arqueología ha sido recientemente aplicada con el fin de evaluar diferentes temas como, por ejemplo, aquellos relaciona- dos con la organización de la tecnología lítica (QUINN, 2015), el comportamiento ritual (MUSCIO, 2002), la caza como estrategia de adquisición de prestigio (HILDEBRANDT \& MC GUIRE, 2003) y la utilización de adornos personales (QUINN, 2006; KUHN, 2014). En líneas generales dicha teoría propone que las señales de comportamientos costosos se relacionan con la transmisión de información honesta que beneficia tanto a los comunicadores como a los destinatarios de estas señales, aun cuando estos sean antagonistas o competidores. La fijación o continuidad de estos comportamientos a largo plazo tiene significado adaptativo y puede devenir en estrategias evolutivamente estables, razón por la que constituyen un indicador de eficacia reproductiva (fitness) (ZAHAVI, 1975; MAYRNARD SMITH \& HARPER, 2003). Dado que las señales costosas pueden beneficiar tanto a los emisores como a los receptores, las interacciones humanas y los comportamientos cooperativos y de reciprocidad (grupales y/o intergrupales) cobran singular importancia dentro de la TSC (cf. BLIEGE BIRD \& SMITH, 2005).

\section{2.- MATERIALES Y MÉTODOS}

Los ornamentos que aquí se describen y analizan provienen de 25 sitios arqueológicos ubicados en diferentes sectores del HPI (Tabla I; Figura 2). La muestra se compone de un total de 348 elementos (Tabla II). Aclaramos que en este trabajo hemos excluido del análisis a los objetos de origen post-hispánico. El relevamiento de la información incluyó los materiales obtenidos en nuestras excavaciones y el registro físico y digital de colecciones museísticas depositadas en el Museo de Ciencias Naturales de La Plata (MLP), National Museum of the American Indian (NMAl), Museo de Ciencias Naturales Carlos Ameghino de Mercedes (provincia de Buenos Aires) y en el Complejo Histórico de Chivilcoy (provincia de Buenos Aires). Se consideraron también los datos publicados por otros autores quienes excavaron parte de los sitios mencionados en la tabla I (TORRES, 1911B; OUTES, 1918; LOTRHOP, 1932; PETROCELLI, 1975; CAGGIANO, 1984) y los generados mediante análisis realizados con dos de las colecciones museísticas mencionadas (BONOMO et al., 2009; BONOMO 2013).

Para la determinación taxonómica de los elementos dentarios y de los moluscos marinos se utilizaron muestras actuales de referencia depositadas en el Museo de Ciencias Naturales Bernardino Rivadavia (ACOSTA et al.,

\begin{tabular}{|c|c|c|c|c|c|c|c|c|c|c|c|c|c|c|c|c|c|c|c|c|c|c|c|c|c|c|}
\hline & $A$ & AR & $\mathrm{Cl}$ & CL & CM & EC3 & Ga & Gz & IL1 & LAN & LB1 & LB2 & LV & PC & RL & CPG & $A^{\circ} S$ & Delta & T 2P & GT 2P & GT 1B & GL G & $\mathrm{BL}$ & $A^{\circ} \mathrm{L}$ & $A^{\circ} \mathrm{F}$ & Total \\
\hline Dientes & 11 & 4 & - & - & - & 6 & 1 & - & - & 1 & - & - & - & - & - & - & - & - & - & 5 & - & - & - & - & 1 & 29 \\
\hline Hueso & $-{ }^{-}$ & - & - & - & - & 1 & - & - & 2 & - & - & - & - & - & - & 1 & - & - & - & 1 & - & - & - & - & - & 5 \\
\hline Lítico & 4 & - & 1 & - & - & - & - & - & & - & 1 & - & 1 & 2 & 2 & - & 1 & - & - & - & - & - & - & 1 & - & 13 \\
\hline Cerámica & - & - & - & 1 & - & - & - & - & - & - & - & - & - & - & - & - & 4 & - & - & - & - & - & - & - & - & 5 \\
\hline Metales & - & - & - & - & - & - & - & - & - & - & - & - & - & - & - & - & - & - & 4 & 4 & 6 & - & - & - & 3 & 17 \\
\hline Valva & 9 & 1 & - & 6 & 8 & 3 & - & 5 & - & - & 1 & 13 & 1 & 8 & - & - & 220 & 2 & - & - & - & 1 & 1 & - & - & 279 \\
\hline Referencias & \multicolumn{15}{|c|}{ inédito, Buc y Caggiano 2015, Acosta et al., 2015} & \multicolumn{6}{|c|}{$\begin{array}{l}\text { Torres 1911b, Lothrop 1932, } \\
\text { Bon et al., 2009, Bonomo } 2013\end{array}$} & \multicolumn{2}{|c|}{ inédito } & $\begin{array}{l}\text { Outes } \\
1918\end{array}$ & $\mid \begin{array}{c}\text { Buc } \\
\text { et al.,. } \\
2014\end{array}$ & 348 \\
\hline
\end{tabular}

Tabla 2: Conjunto de artefactos ornamentales analizados en el trabajo. Los sitios $A^{\circ} \mathrm{L}$ y $A^{\circ} \mathrm{F}$ corresponden con depósitos guaraníes. / The set of ornamental artefacts analysed in the work. Sites $A^{\circ} \mathrm{L}$ and $\mathrm{A}^{\circ} \mathrm{F}$ correspond to Guarani deposits. 
2014, 2015). Se realizó el relevamiento morfométrico y estilístico de las piezas. Para determinar la composición de metales y rocas se realizaron microanálisis por dispersión de energía de rayos- $X$. Los rastros de uso y manufactura de las piezas se identificaron microscópicamente con lupa binocular (entre 10 y 50X) y microscopio metalográfico (entre 50 y 200X).

\section{3.- RESULTADOS}

\subsection{Dientes}

La colección está compuesta por 30 elementos dentarios que provienen de los sitios Anahí, El Cazador 3, Las Ánimas, Arroyo Fredes, La Argentina y Garín (ver Tabla II; Figura 3). En las colecciones del MLP de Túmulo II del Paraná Guazú hemos reconocido 5 caninos, todos ellos correspondientes a Felis onca (L. SOILBELZON com. pers. 2014). En dicho sitio (1911b) menciona que recuperó "...más de treinta molares y caninos de este felino (tigre) (...) uno de los incisivos presenta una cintura que parece haberse destinado a la aplicación de una cuerda de suspensión" (TORRES, 1911b: 251).

Dentro de los conjuntos analizados existe un evidente predominio de los caninos pertenecientes a distintas especies de carnívoros. Los cánidos dominan la muestra con ejemplares de Chrysocyon brachyurus (aguará guazú), Dusicyon avus (zorro extinto) y Canis familiaris (perro doméstico). Los asignados a zorros de pequeño tamaño no pudieron ser diferenciados específicamente, por lo que podrían corresponder a Lycalopex gymnocercus (zorro gris) o bien a Cerdocyon thous (zorro de monte) (ver ACOSTA et al., 2014). Los félidos están representados por Puma concolor (puma) y Panthera onca (yaguareté), los mustélidos, por Lontra longicaudis (lobito de río) y los procyónidos, por Nasua nasua (coatí). Completa la muestra un segundo molar de Hydrochaeris hydrochaeris (carpincho) modificado que puede interpretarse como un adorno facial, sea labial, orejera, o nariguera. La pieza fue abradida

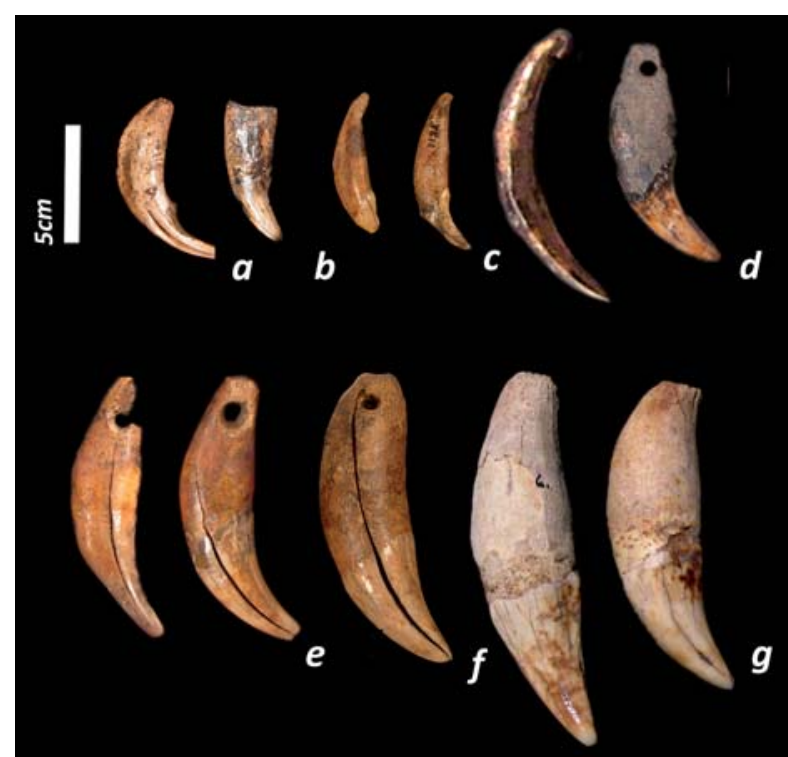

Fig. 3. Dientes de carnívoros: a) Aroyo Fredes, b) Anahí, c) La Argentina, d) Anahí, e) El Cazador 3, f) La Argentina g) "Brazo Largo" MLP Col. Torres. / Carnivore teeth: a) Aroyo Fredes, b) Anahí, c) La Argentina, d) Anahí, e) El Cazador 3, f) La Argentina g) "Brazo Largo" MLP Torres Coll.

y pulida, desgastando los primas y el cemento asociado a los fléxidos internos. Esto da un aspecto de "incisión" en la vista frontal del artefacto (Figura 4).

La mayoría de los caninos fueron perforados en la raíz sobre el extremo superior $(n=17)$, lo cual sugiere que fueron utilizados como pendientes. Los orificios son mayormente bicónicos (realizados posiblemente desde una cara y finalizados perforando ambas) y con un rango de tamaño de entre 1,5 y 3,5 mm (ACOSTA et al., 2014). El resto de las piezas no presentan modificaciones antrópicas a ojo desnudo. Sin embargo, en cuatro de ellas (sobre 6 analizadas $^{1}$ ), se registraron huellas microscópicas de corte y raspado de las superficies que podrían ser el resultado de su preparación, manufactura o producto de su uso

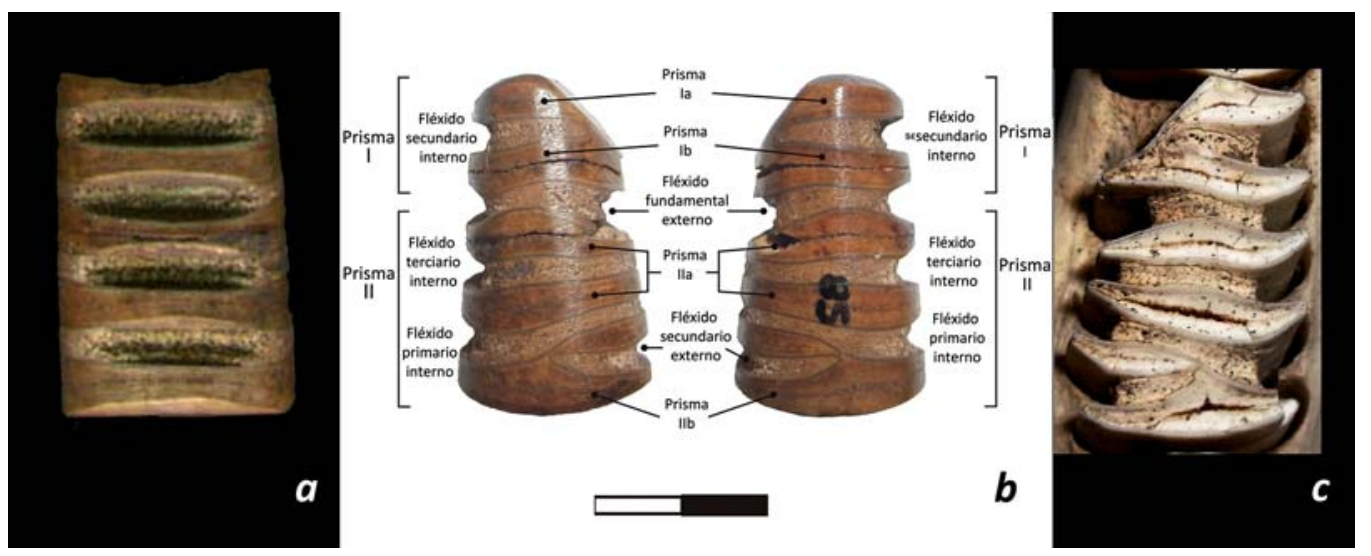

Fig. 4. Artefacto elaborado sobre un molar de $\mathrm{H}$. hydrochaeris: a) vista frontal del artefacto, b) vista lateral del artefacto, c) vista cenital de un segundo molar inferior actual de $\mathrm{H}$ hydrochaeris. / Tool made on $\mathrm{H}$. hydrochaeris molar: a) artifact's frontal view; b) artifact's lateral view, c) 2nd inferior molar, cenital view.

\footnotetext{
${ }^{1}$ No se analizaron en microscopio las piezas de la colección Torres del MLP. Tampoco se incluye una pieza de Anahí y otra de Garín.
} 
(ACOSTA et al., 2014). Es posible que estas últimas hayan sido preformas para la confección de pendientes o piezas que fueron sujetadas o enlazadas de un modo diferente de las que presentan perforaciones (ver también TORRES 1911b: 251).Si bien la mayoría de los elementos han sido recuperados en áreas de actividades múltiples, existen al menos dos sitios (Garín y La Argentina), en donde se registraron caninos sueltos (con y sin perforación) utilizados a modo de acompañamiento mortuorio (ver detalles en ACOSTA \& MAZZA, 2015).

\section{2. Óseo}

Los adornos en hueso están representados por cuatro elementos, lo que es llamativo frente a la gran cantidad de artefactos óseos que se han reconocido en la mayoría de los depósitos arqueológicos del HPI (BUC, 2012). Se identificó una cuenta tubular en el sitio El Cazador 3 y dos pendientes de sección plana en Isla Lechiguanas 1 (Figura 5a). El primero (IL120) es un pequeño fragmento de hueso indeterminado, cuya superficie fue pulida y sobre la cual se realizó una perforación y una pequeña hendidura en el extremo superior; fue recuperado en el nivel cerámico de este sitio (Figura 5b). El segundo (IL35) presenta dos orificios, uno de ellos fracturado; el nivel de su procedencia es indeterminado (Figura 5c; ver BUC \& CAGGIANO, 2015). Ambos elementos, por su sección plana y perforaciones, tienen cierta similitud con una pieza publicada por Torres (1911b: 283) en el sitio Cementerio 1 del
Paraná Guazú, definida como un posible amuleto (ver Figura 5d). El elemento tiene una perforación superior y una fractura lateral que parece estar atravesando una segunda perforación inferior de mayor tamaño. En una de sus caras posee tres campos separados por líneas transversales y numerosos cortes de menor tamaño perpendiculares que, de acuerdo al propio Torres "...tratan de interpretar los contornos de la figura humana." (TORRES, 1911b: 283).

\subsection{Material lítico}

Se recuperaron 11 cuentas líticas en los sitios: Cerro de los Indios, Las Vizcacheras, La Bellaca 1, Punta Canal, Anahí y Río Luján (Figura 6). Todas ellas elaboradas sobre las genéricamente denominadas "rocas verdes". El análisis de las potenciales fuentes de estas materias primas se encuentra en curso, pero en principio notamos que el conjunto está formado por variantes de silicatos, no estando representadas aquellas que contienen cobre (malaquita, turquesa, crisocola) salvo en un caso (Punta Canal; BUC et al. no publ.). Esto sugiere que para la elaboración de las cuentas se utilizaron diferentes tipos de rocas, posiblemente provenientes de más de un lugar o región (ver más abajo). Morfológicamente, con la excepción de una pieza de Río Luján, que es tubular, las cuentas son todas discoidales, ya sea circulares, subcirculares, cuadrangulares e irregulares (Figura 6). Las perforaciones se encuentran generalmente en el sector central de las piezas, aunque en algunos casos están ligeramente desplazadas hacia un ex-
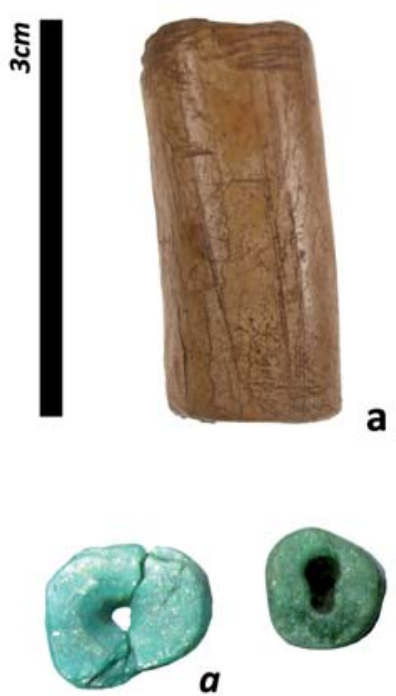

$a$
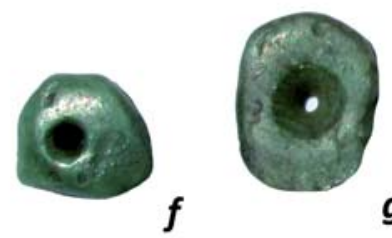

g
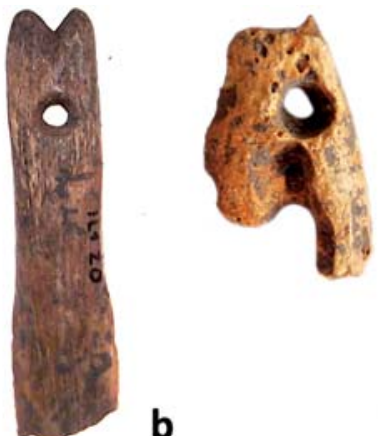

b
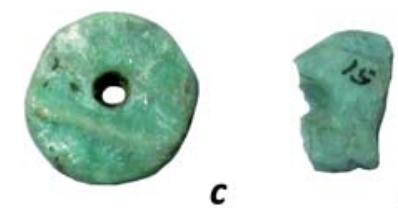

c
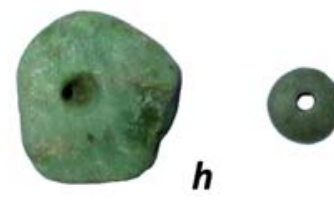

c

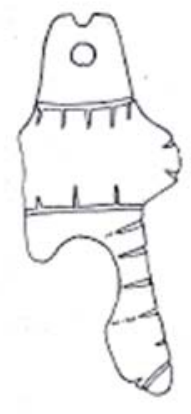

d

Fig. 5. Adornos óseos: a) cuenta tubular (EC3 81), b) pendiente de sección plana (IL 20), c) pendiente de sección plana (IL 35), d) pendiente tomado de Torres, 1911: fig. 102. / Bone ornaments: a) tubular bead (EC3 81), b) flat cross-section pendant (IL 20), c) flat cross-section pendant (IL 35), d) pendant taken after Torres, 1911: fig. 102.

Fig. 6. Cuentas sobre rocas verdes: a) Cerro de j los Indios, b-d) Anahí, e) Río Luján, f) Punta Canal, g) La Bellaca 1, h) Anahí, i) Las Vizcacheras. I Beads on green rocks: a) Cerro de los Indios, b-d) Anahí, e) Río Luján, f) Punta Canal, g) La Bellaca 1, h) Anahí, i) Las Vizcacheras.
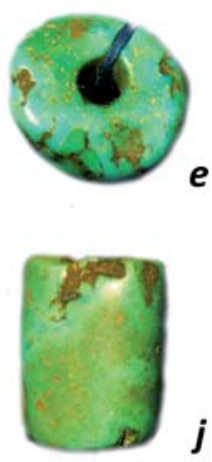
tremo. La muestra tiene gran heterogeneidad en los tamaños, así como también en el diámetro y tipos de orificios.

A los casos mencionados se suma una pieza diferente, identificada por LOTHROP (1932) en Arroyo Sarandí. Se trata de un disco lítico de color púrpura que según el autor habría sido utilizado como orejera. Dicha pieza sería similar a las descriptas por Lehmann-Nitsche para sitios del norte de la provincia de Río Negro (ver LOTHROP, 1932: 177).

Como caso excepcional debe mencionarse parte de un tembetá confeccionado sobre cuarzo, proveniente del sitio guaraní Arroyo Largo (OUTES, 1918: 173, figura 29). El fragmento presenta una estrecha similitud con los tembetás recuperados en otras regiones y sitios atribuidos a la tradición tupiguaraní. Dichos adornos se caracterizan por presentar una espiga tubular alargada, cuyo sector proximal tiene forma de T. Si bien habrían sido preferentemente elaborados en cuarzo, también se utilizaron otras materias primas como amazonita, hueso y resina (AMBROSETTI, 1895; MENGHIN, 1957; PROUS, 2011; LOPONTE \& ACOSTA, 2013). De hecho, en una de las colecciones del MLP identificada como "Delta del Paraná" (sin procedencia concreta) se ha registrado una pieza completa sobre resina (BONOMO et al., 2009) morfológicamente idéntica a las manufacturadas en cuarzo halladas en otros sitios guaraníes (ver comparación en Figura 7).

\subsection{Cerámica}

Se contabilizó un total de seis adornos cerámicos provenientes de los sitios Cerro Lutz y Arroyo Sarandí. En el primero de ellos se identificaron dos elementos decorados por surco rítmico, representados por una cuenta de forma ovoide perforada en sus extremos y con motivos romboidales (Figura 8a), y una delgada placa de forma subrectangular, con incisiones en forma de grecas (Figura 8b). En $A^{\circ}$ Sarandí se registró un pendiente subrectangular con perforación superior y decorado en ambas caras mediante líneas continuas en zig-zag formando un motivo de características zoomorfas similares a las de un ofidio (Figura 8c).

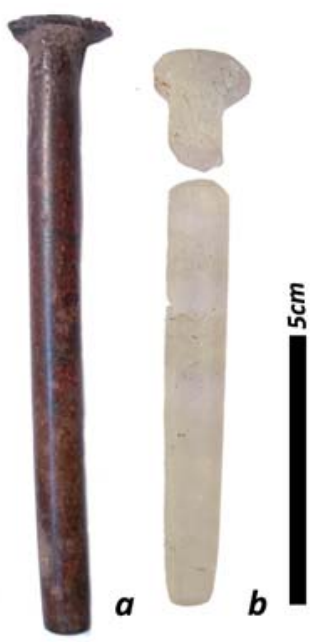

Fig. 7. Tembetas guaraníes: a) tembetá de resina registrado como "Delta del Paraná" en la colección Torres MLP, b) fragmentos de tembetá de cuarzo recuperado en el sitio Corpus, provincia de Misiones (Argentina). Tomado y modificado de Loponte \& Acosta (2013). / Guaraní tembetá: a) tembetá made on tree gum recorded as "Delta del Paraná" in Torres Collection $M L P$, b) fractured tembetá on quartz from the Corpus archaeological site, Misiones (Argentina). Taken and modified after Loponte \& Acosta (2013).
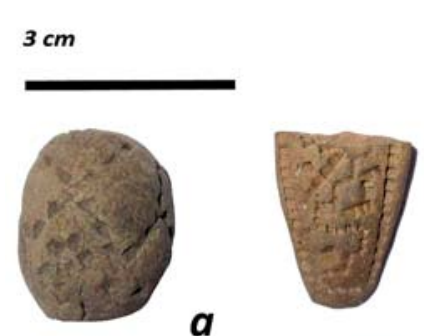

$\boldsymbol{a}$

b
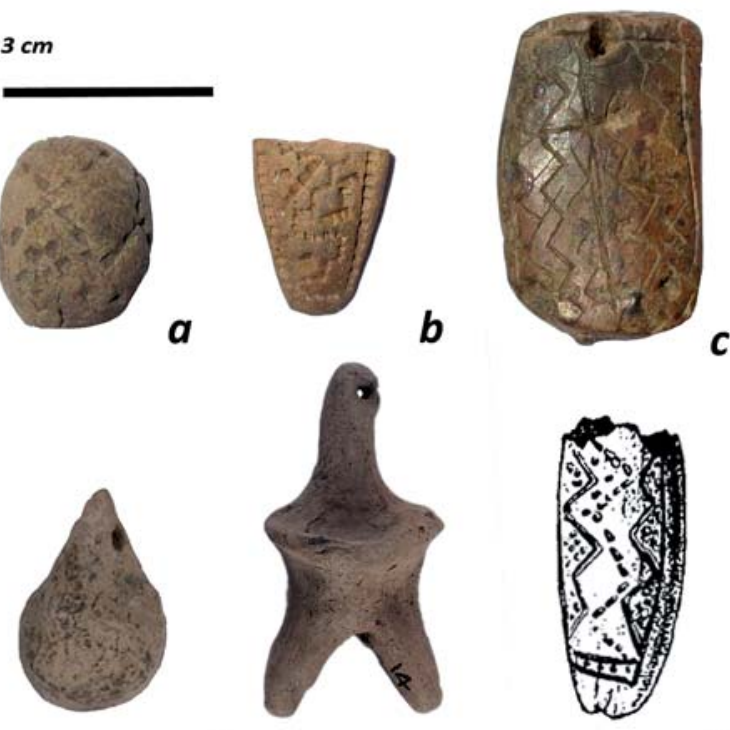

$\boldsymbol{d}$

e

$f$

Fig. 8. Ornamentos en cerámica: a) cuenta Cerro Lutz, b) placa decorada Cerro Lutz, c) pendiente Arroyo Sarandí, d) cuenta Arroyo Sarandí (NMAl 144785), e) figura antropomorfa recuperada en Arroyo Sarandí (NMAl 144786), f) placa decorada Arroyo Sarandí tomada de Lothrop 1932: Fig. 78c (Lothrop, 1932:172). / Ceramic ornaments: a) bead from Cerro Lutz, b) decorated plaque from Cerro Lutz, c) pendant from Arroyo Sarandí, d) bead from Arroyo Sarandí (NMAl 144785), e) anthropomorphic figure from Arroyo Sarandí (NMAI 144786), f) decorated plaque from Arroyo Sarandí taken after Lothrop 1932: Fig. 78c (Lothrop, 1932: 172).

Las tres piezas restantes fueron recuperadas por LOTHROP (1932): la primera es de forma ojival (Figura 8d) y la segunda se encuentra representada por un modelado antropomorfo, ambas están sin decorar pero presentan perforaciones en su extremo superior (Figura 8e). La última es una placa fragmentada de tamaño pequeño, subrectangular, decorada por incisión mediante zig-zags; morfológica y estilísticamente similar a la de Cerro Lutz (Figura 8f).

\subsection{Metales}

Los objetos metálicos se encuentran representados por 17 elementos que han sido identificados tanto en ocupaciones de origen guaraní como en sitios de cazadoresrecolectores locales.

En los sitios generados por cazadores-recolectores, Torres fue uno de los primeros en advertir la presencia de objetos metálicos. En Túmulo 1 del Brazo Gutiérrez recuperó "... una pequeña lámina de cobre de forma rectangular, adherida al cráneo número 29; lleva en la proximidad de uno de sus contornos un pequeño agujero" (TORRES, 1911b: 351). La colección de la piezas recuperadas por Torres, actualmente depositadas en el MLP, fueron re-analizadas por BONOMO et al. (2009). La muestra está compuesta por 14 objetos provenientes de los sitios Túmulo I del Brazo Gutiérrez y Túmulo I y II del Paraná Guazú. Se reconocieron cuentas cuadrangulares de bordes redondeados con perforación, fragmentos y láminas. Si bien estos materiales fueron mencionados por Torres (1911b), algu- 
nos de ellos permanecían inéditos (BONOMO et al., 2009: 81). Asimismo, Torres (1911b) señaló que parte de estos artefactos se hallaban asociados a entierros humanos, dado que "... se las ha encontrado casi adheridas a los cráneos, pues han dejado su señal en el hueso, debido a la fuerte oxidación..." (Torres, 1911b:258). De acuerdo a los estudios composicionales publicados (TORRES, 1911b; BONOMO et al., 2009) sabemos que fueron elaborados sobre cobre, siendo muy posible que provengan de la región andina (AMBROSETTI, 1905; TORRES, 1911b; LOPONTE, 2008, BONOMO et al., 2009). El origen prehispánico de todos estos objetos se encuentra avalado por los fechados que se disponen para los sitios mencionados (Tabla I).

En el sitio guaraní Arroyo Fredes se recuperaron tres elementos, uno de ellos es una cuenta o disco hecho de cobre, otro un fragmento de lámina de cobre, y el tercero un posible alfiler o "topu" fracturado de bronce con componentes de estaño y cinc (Cu 95,56\%; Sn 1,25\%; Zn 3, 10 $\%$; Figura 9; ver BUC et al., 2013). Los dos primeros, tanto por su morfología como por su composición química, serían originarios de la región andina como se ha propuesto tradicionalmente (ver arriba). Sin embargo, la tercera pieza merece una apreciación diferente. En principio, el diseño no contempla similitudes con los topus inkaicos que mantienen formas discoidales y semilunares (e.g. REICHLEN, 1940). Solo hemos documentado la existencia de una pieza con parecido estilístico en un sitio de contacto hispano-indígena situado en la provincia de Santiago del Estero, al norte del área de estudio (cf. GRAMAJO DE MARTÍNEZ MORENO, 1979). A su vez, la presencia de cinc hace suponer que la aleación podría ser de origen post-hispánico, aunque los registros publicados para estas piezas tienen porcentajes de cinc significativamente mayores al de Arroyo Fredes (>25\%; ver por ejemplo DEBENEDETTI, 1911; GLUZMAN \& GONZÁLEZ 2009; TAPIA et al., 2009). Debe tenerse en cuenta que los fechados obtenidos para este sitio lo ubican en momentos inmediatos a la conquista europea (Tabla I). Cabe mencionar por último que los tres elementos fueron identificados en contextos de actividades múltiples.

\subsection{Material malacológico}

Los moluscos fueron una de las principales materias primas utilizadas para la elaboración de adornos. La muestra analizada está compuesta por 279 piezas. Los soportes utilizados para su confección incluyen tanto especies de origen local (fluviales) como foráneo (marinas). Entre las primeras, se destacan las valvas de Diplodon sp., aunque no desestimamos que se hayan empleado otras especies disponibles localmente (e.g. Ampullaria sp.). Debe señalarse que los moluscos dulceacuícolas constituyeron un recurso predecible y abundante en el área de estudio, conformando parte de la dieta de las poblaciones prehispánicas. Si bien su contribución en biomasa habría sido más bien marginal, hacia fines del Holoceno tardío comenzaron a ser intensamente explotados (ver ACOSTA,

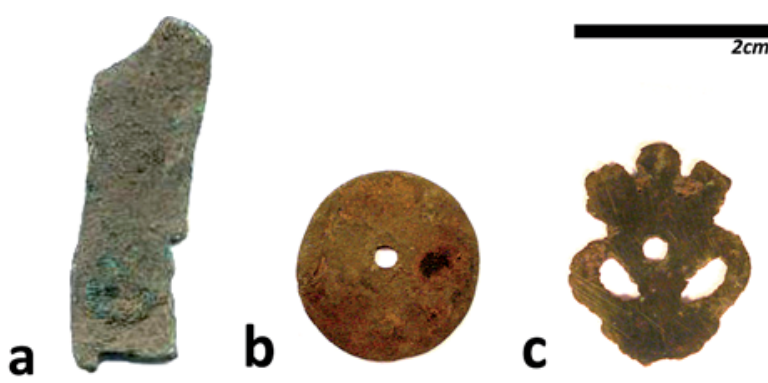

Fig. 9. Artefactos de metal recuperados en el sitio Arroyo Fredes: a) placa, b) disco, c) alfiler o topu. / Metal tools recovered from Arroyo Fredes: a) plaque, b) disc, c) pin or topu.

2005; LOPONTE, 2008, ACOSTA et al. 2010). De hecho se han registrado sitios en donde se observan acumulaciones de Diplodon sp. densas y concentradas formando verdaderos concheros (e.g. ACOSTA et al., 2010), lo cual también es un indicador del alto grado de disponibilidad que tuvo esta especie como materia prima para la elaboración de artefactos.

Dentro de los conjuntos analizados predominan las cuentas. Se registraron 270 ejemplares, de los cuales 220 corresponden a un collar recuperado por Lothrop (1932) en el sitio Arroyo Sarandí (BONOMO, 2013). Dicha pieza, depositada actualmente en el NMAI, fue elaborada con gasterópodos marinos asignados a Urosalpinx haneti (cf. BONOMO, 2013). Todos ellos fueron intencionalmente perforados y se hallaron asociados a un entierro femenino a modo de acompañamiento mortuorio (LOTHROP, 1932). En la colección Gaggero y Fernández (1928, MLP), perteneciente al sitio Brazo Largo, se reconoció un ejemplar de U. haneti con una perforación morfológicamente similar a las registradas en Arroyo Sarandí (ACOSTA et al., 2015; Figura 10b). En este sitio también se identificaron unas diez cuentas discoidales, originalmente consignadas como "discos perforados" (ver LOTHROP, 1932: 177; Figura 10a) que corresponderían a Glycymeris longior (BONOMO, 2013).

El resto de las cuentas fueron elaboradas sobre valvas de moluscos fluviales. En Anahí, Punta Canal, Guazunambí, La Bellaca 2, Cerro Lutz y La Argentina fueron identificadas en contextos domésticos. Como caso excepcional debe mencionarse que en el sitio Cerro Mayor se encontraban asociadas a una estructura funeraria (ACOSTA et al., 2013). Dentro del conjunto total de cuentas, la forma más frecuente es la discoidal. El 90,3\% de las mismas presenta el contorno regularizado y perforación desplazada del centro. En Punta Canal se recuperaron cuatro ejemplares discoidales sin perforación, que muy posiblemente corresponden a preformas. A nivel intersitio se registra algún grado de heterogeneidad en el tamaño y forma de las cuentas. Por ejemplo, en Anahí y Punta Canal predominan las formas discoidales de tamaño mediano con perforación desplazada del centro de la pieza. Las cuentas de Cerro Mayor tienen estas mismas características, pero con la particularidad de que 


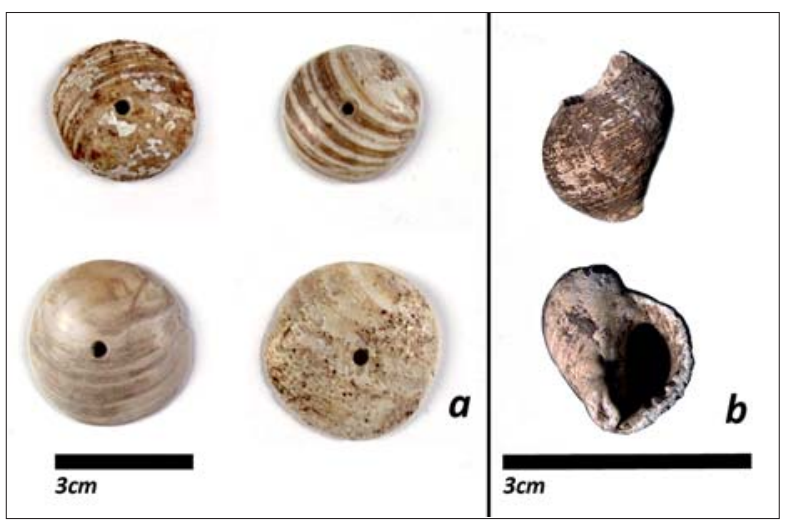

Fig. 10. Elementos sobre valvas de moluscos marinos: a) ejemplares de Glycymeris longior con perforaciones recuperados por Lothrop (1932) en el sitio Arroyo Sarandí (NMAl 144778.000), b) Vista dorsal y ventral del ejemplar de Urosalpinx haneti procedente del sitio Brazo Largo (colección Gaggero y Fernandez 1928, MLP). Tomado y modificado de Acosta et al. no publ. / Items on marine mollusks: a) Glycymeris longior perforated ítems recovered by Lothrop (1932) in Arroyo Sarandí (NMAl 1474778.000), dorsal and ventral view form Urosalpinx hanetispecimen recovered in Brazo Largo (Gaggero y Fernández Collection 1928, MLP). Taken and modified after Acosta et al. unpublished.

todas conservan parte de la rebarba natural de la valva en el sector opuesto a la perforación. A diferencia de las anteriores, la mayoría de las cuentas de La Bellaca 2 tienen forma de anillo y son de menor tamaño (ver Figura 11). Además, en la colección Torres del MLP se encuentran dos discos de gran tamaño con perforación central (BONOMO et al., 2009; Figura 12), uno de ellos con pequeñas incisiones alrededor del borde exterior.

Sobre valvas de moluscos fluviales también se confeccionaron tembetás. Un total de nueve ejemplares fueron identificados en Las Vizcacheras, Punta Canal, El Cazador 3, Cerro Lutz, Guazunambí y La Bellaca 1 (LOPONTE, 2008). Todos presentan una forma en "T" constituida por un eje vertical y otro transversal en el extremo superior. Solo dos tembetás presentan el eje transversal completo, ya que en la mayoría de los casos uno de los lados se encuentra fracturado. Si bien todas las piezas son morfológicamente similares, presentan cierta varia-

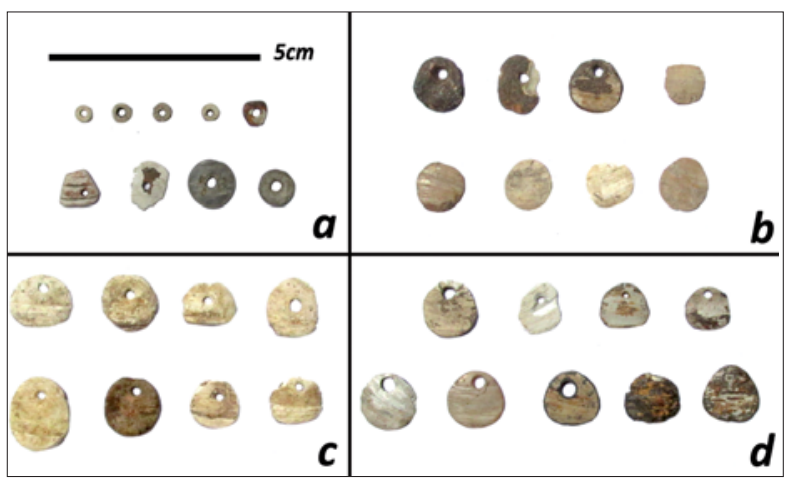

Fig. 11. Cuentas en valva de moluscos de agua dulce: a) La Bellaca 2, b) Punta Canal, c) Cerro Mayor, d) Anahí. / Beads on fresh water mollusks: a) La Bellaca 2, b) Punta Canal, c) Cerro Mayor, d) Anahí.

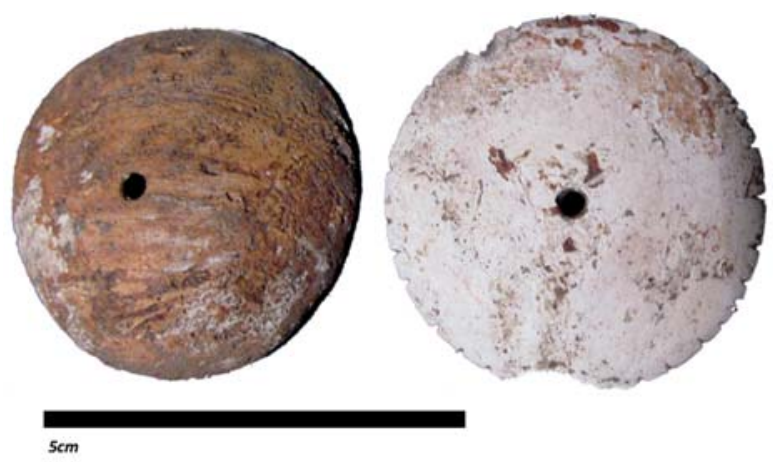

Fig. 12. Discos perforados procedentes de la colección Torres, MLP. / Perforated discs from Torres Collections, MLP.

bilidad. Por ejemplo, en La Bellaca 1 y en Las Vizcacheras el eje transversal y el vertical son prácticamente iguales, mientras que en los demás casos el eje central es más largo y delgado (Figura 13). Cabe agregar que el ejemplar hallado en Punta Canal se destaca por ser el más pequeño del conjunto.

Finalmente, también se han identificaron exoesqueletos de moluscos marinos sin modificación antrópica, aunque en bajas proporciones. Es posible que estas piezas hayan sido conservadas como reservorio de materia prima o por algún tipo de valoración simbólica particular. Tal es el caso de un ejemplar de Olivancillaria urceus registrado en el sitio Brazo Largo (GAGGERO \& FERNÁNDEZ 1928, $\mathrm{MLP}$ ). Dentro de la misma situación se encuentra un cirripedio (Coronula diadema) identificado en el sitio La Glorieta (colección Castro 1925, MLP, para más detalles ver ACOSTA et al., 2015).
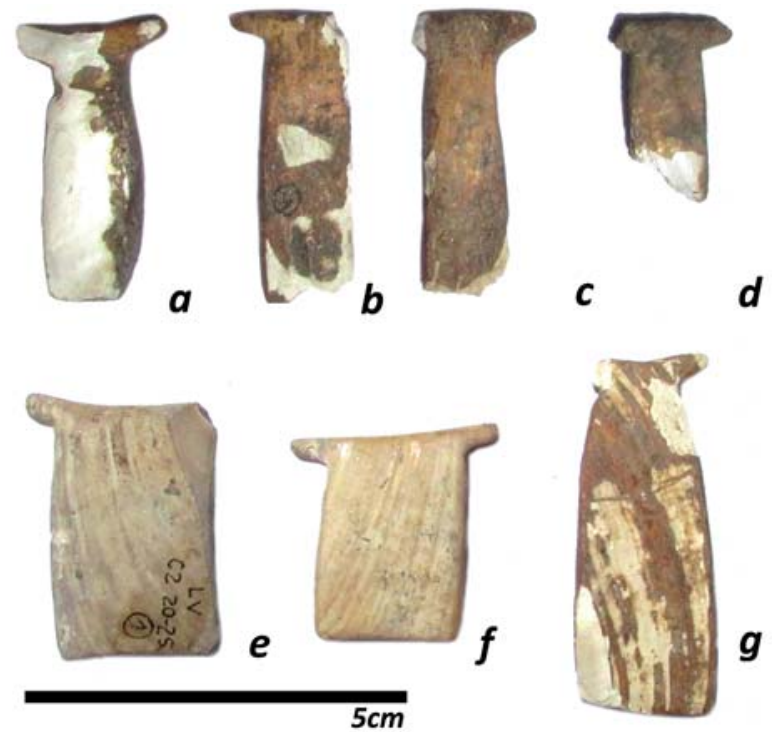

Fig. 13. Tembetás en valva: a) El Cazador 3 , b) Guazunambí, c) Cerro Lutz, d) Punta Canal, e) Las Vizcacheras, f) La Bellaca 1, g) Cerro Lutz. ( Shell tembetá: a) El Cazador 3, b) Guazunambí, c) Cerro Lutz, d) Punta Canal, e) Las Vizcacheras, f) La Bellaca 1, g) Cerro Lutz. 


\section{4.- DISCUSIÓN}

Los grupos humanos del HPI utilizaron una amplia gama de objetos ornamentales elaborados sobre diferentes materias primas. A pesar de que la mayoría son elementos colgantes, se observa una significativa diversidad en cuanto a sus formas y/o improntas estilísticas. Los cronistas del siglo XVI han efectuado distintas referencias sobre el uso de adornos (fundamentalmente faciales) entre las poblaciones nativas. Entre aquellas de carácter más general pueden citarse las de Ulrico Schmidel y Luis Ramírez:

"Los timbú derivaban su sobrenombre de los adornos que se ponían en las narices, y fueron los guaraní quienes se lo aplicaron, como que por éstos fue por lo que los españoles conocieron a aquéllos" (SCHMIDeL, [1567] 1881:60-61).

"En la comarca de la dha fortaleza ay otras naciones las quales son carcarais y chanaes y beguas y chanaestinbus y tinbus (...) tienen todos oradadas las narizesansionbres como mujeres por tres partes y las orejas. Los onbres oradan los labios por la parte baja destos..." (RAMÍREZ, [1528] 2007: 31).

A partir de los análisis aquí efectuados se observa la existencia de dos grandes grupos de objetos: los elaborados sobre materias primas locales y los producidos sobre materiales exóticos. En términos de señalización costosa, entendemos que ambos fueron en todo caso portadores de señales honestas, tanto de bajo como alto costo (cf. KUHN, 2014).

\subsection{Adornos sobre materias primas locales}

Dentro de adornos sobre materias primas locales se destacan las cuentas confeccionadas sobre exoesqueletos de moluscos fluviales. Con excepción del sitio Cerro Mayor, en donde estaban asociadas a restos humanos, provienen siempre de contextos domésticos. Además, si bien hay cierta homogeneidad en tamaño y forma (cuentas medianas discoidales), se observan particularidades en algunos conjuntos. Como mencionamos, en Anahí y Punta Canal las cuentas presentan una significativa similitud siendo distintas de las recuperadas en Cerro Mayor, y más aún de las identificadas en La Bellaca 2. Estas propiedades podrían estar vinculadas con la destreza del artesano pero no descartamos la existencia de un concepto técnico intragrupal en el "modo de hacer" estos adornos.

También vimos que los moluscos fluviales se utilizaron para la manufactura de los tembetás, lo cuales a juzgar por la recurrencia y estandarización de su diseño debieron tener una particular connotación (social y/o simbólica) para sus usuarios y grupo de pertenencia. Hasta ahora todos fueron hallados en áreas domesticas y si bien comparten un patrón morfológico similar, detectamos ciertas variaciones. Como mencionamos, en función de su tamaño y diseño (relación eje vertical-transversal) podemos separar las piezas de Las Vizcacheras y La Bellaca 1 del resto de los conjuntos analizados. Las diferencias de tamaño observa- das podrían estar relacionadas con la edad de los usuarios. LOPONTE (2008) señaló que los tembetás son concurrentes con otros rasgos arqueológicos, en especial, su presencia en contextos arqueológicos que tienen un evidente predominio de la alfarería lisa. De hecho, de los seis sitios donde se recuperaron tembetás, solamente Punta Canal tiene cerámica decorada. En términos morfológicos y estilísticos, estos adornos labiales, constituyen un rasgo distintivo de los grupos cazadores-recolectores que ocuparon la baja cuenca del Paraná (LOPONTE, 2008), siendo diferentes de los que usaban los grupos guaraníes (ver más arriba). Etnográficamente, sabemos que entre estos últimos el tembetá se vinculaba con el pasaje de los individuos de la infancia a la pubertad, constituyendo un símbolo típico de masculinidad (MÜLLER, 1989).

Los ornamentos locales posiblemente se utilizaron para transmitir información emblemática dentro del grupo de pertenencia, como una forma de demarcación etaria, social y/o étnica. En un sentido más general, habrían funcionado como medios de comunicación, cuyo fin habría sido promover acciones compartidas y reforzar lazos sociales relacionados con la identidad y la cooperación entre los individuos dentro de un mismo grupo. Esto de algún modo garantiza saber quién es quién en contextos de creciente densidad poblacional y en donde los individuos comparten intereses similares, condición bajo cual la transmisión de señales honestas es más fácil de mantener (KUHN, 2014)

Por otra parte, los adornos elaborados sobre arcilla y hueso son los que menor frecuencia tienen dentro de los elementos de origen local, lo cual es sorprendente frente al amplio dominio tecnológico que existió de la alfarería y la tecnología ósea local (ACOSTA, 2005; LOPONTE, 2008; PÉREZ, 2010; BUC, 2012). La escasa presencia de ornamentos cerámicos y óseos no se relacionaría con deficiencias en el muestreo de los sitios, sino que estaría vinculada con algún tipo de decisión cultural. La singularidad de estos objetos, junto a su escasa representación, sugiere que pudieron haber tenido algún significado o vaIoración especial para aquellos que los utilizaron, además de un alto poder de señalización, dado que cuanto más abundantes son las señales materializadas, menor será la potencia de las mismas (cf. QUINN, 2015).

Los últimos elementos locales utilizados como ornamentos son los dientes de mamíferos. Como caso excepcional, vimos que en el sitio Anahí se registró un molar de carpincho $(H$. hydrochaeris) totalmente modificado, que podría haberse utilizado como adorno facial (cf. Loponte, 2008). En el área de estudio la escasa a nula frecuencia de esta especie dentro de los conjuntos arqueofaunísticos ha sido vinculada con la existencia de algún tipo de restricción o tabú alimenticio (SALEMME, 1987; ACOSTA, 2005), por lo cual la confección de dicho adorno pudo tener algún tipo de connotación simbólica o ideológica en particular. El resto de los pendientes fueron elaborados sobre caninos de distintas especies de carnívoros. Si bien la mayoría fueron recuperados en contextos domésticos, en los sitios La Argentina y Garín se hallaban asociados a entierros pri- 
marios (LOPONTE, 2008; ACOSTA \& MAZZA, 2015). Cabe agregar que en algunos contextos funerarios del HPI, además de dientes, se han registrado cráneos de carnívoros (e.g. P. onca y Dusicyon avus), lo cual sugiere que estos últimos constituyeron parte de las esfera simbólica de los cazadores-recolectores locales (ver ACOSTA, 2005; ACOSTA \& MAZZA, 2015).

Por otro lado, debe señalarse que en el HPI la gran mayoría de las inhumaciones humanas carecen de acompañamiento mortuorio, aspecto que ha sido vinculado con la ausencia de jerarquías sociales. Si bien la existencia de desigualdad institucional es una hipótesis plausible, aún no ha podido ser contrastada positivamente (ver discusión en LOPONTE, 2008; MAZZA \& LOPONTE, 2012). De no haber existido en el HPI inequidad social ¿que otros factores podrían dar cuenta del entierro de unos pocos individuos con restos de carnívoros? En trabajos anteriores hemos planteado algunas posibles explicaciones al respecto (ACOSTA, 2005; ACOSTA \& MAZZA, 2015). A partir de analogías etnográficas, señalamos la importancia que suelen adquirir algunos carnívoros dentro de los sistemas simbólicos de numerosos grupos de cazadores-recolectores actuales. Parte de esta simbología descansa sobre creencias animistas que vinculan su poder predatorio con el éxito en las actividades cinegéticas. Debido a esto, en distintos grupos se ha documentado la utilización de ciertas partes esqueletarias como amuletos, es decir como elementos que poseen propiedades mágicas que pueden favorecer o propiciar la caza, actuar por sus efectos medicinales o brindar protección (e.g. TORRES, 1911A, 1911B; HILL, 2013; ACOSTA ET AL., 2014). Al respecto, TORRES (1911b), refiriéndose a los hallazgos arqueológicos de dientes de carnívoros en el Delta del Paraná señaló que "Hasta hace muy poco los habitantes de esta misma comarca usaban un colmillo de tigre colgado del cuello como talismán contra las enfermedades, o como porte bonheur en las cacerías." (TORRES, 1911b: 251).

Diversas referencias de sociedades cazadoras-recolectoras señalan la importancia que tiene la caza como actividad masculina y el prestigio social que reciben aquellos que se transforman en cazadores exitosos (e.g. HILL \& HURTADO, 1996; HAWKES et al., 1997; BLIEGE BIRD et al., 2001). Es importante mencionar que en el HPI, hasta el momento, los restos de carnívoros hallados en estructuras funerarias se encuentran únicamente asociados a individuos masculinos adultos (ver ACOSTA \& MAZZA, 2015). La adquisición de prestigio a través de la caza y el reconocimiento social que reciben los cazadores que son exitosos constituyen parte de las señales costosas que posibilitan promover conductas altruistas al brindar información sobre la calidad o habilidades que tienen ciertos individuos (cf. ZAHAVI, 1975). Además, en el marco de la ecología del comportamiento, entendemos que los beneficios sociales que obtienen estos últimos pueden incrementar la eficacia reproductiva (SMITH et al., 2003). En síntesis, una de las posibles interpretaciones o hipótesis sobre la relación humanos-carnívoros que se observa en el HPI es que dicho fenómeno se relacione con señales cos- tosas vinculadas a la caza. La materialización de estas conductas podría haber estado relacionada con la utilización de cráneos y dientes (con o sin modificación) como amuletos. También es también posible que la presencia de los carnívoros en los contextos funerarios haya tenido algún tipo de componente ritual (ACOSTA et al., 2014) destinado a fortalecer la cooperación intra-grupo (sensu SOSIS \& ALCORTA, 2003: 267-268).

A pesar de estas excepciones vimos que la mayoría de los adornos fueron hallados en espacios domésticos, por ende vinculados a las actividades cotidianas de sus usuarios, lo cual es esperable para una señal de bajo costo (cf. KUHN, 2014). Además, es muy posible que su ingreso al registro se relacione con su pérdida o descarte por rotura (SCHIFFER, 1976). Una situación diferente ocurre con los objetos compuestos (e.g. abalorios), que suelen ser recuperados frecuentemente en estructuras mortuorias. Los ornamentos locales fueron, posiblemente, producidos y utilizados dentro de las mismas unidades sociales. De hecho se han encontrado preformas de cuentas de valva como es el caso de Punta Canal. Esto no excluye la posibilidad de que algunos elementos fueran obtenidos a través del intercambio entre bandas locales que compartiesen códigos o señales similares en cuanto a la información que éstos debieron contener. En algunos grupos etnográficos actuales como los Kung! es común que se utilicen e intercambien cuentas de valva como regalos a través del denominado hxaro (WIESSNER, 1984, 2002). Esta conducta se observa fundamentalmente entre las mujeres, las cuales invierten energía en la confección de abalorios con el deseo de impresionar al sexo opuesto y reforzar las relaciones de reciprocidad entre diferentes bandas (WIESSNER 1984: 204).

\subsection{Ornamentos sobre materia prima alóctona}

Hemos visto que las piezas de origen exótico fueron elaboradas en metal, moluscos marinos y rocas verdes. Si bien aún no está del todo claro cuáles fueron los lugares de procedencia de estas últimas, es evidente que no son locales. Si bien parte de ellas puede provenir de un área relativamente cercana como la República Oriental del Uruguay (ver BAEZA, 2013) donde existen claras vinculaciones culturales con los diferentes sectores que comprende el HPI (e.g. SERRANO, 1931, 1972; FIGUEIRA, 1965; CAGGIANO, 1984), es posible también que provengan de zonas más alejadas como, por ejemplo, las Sierras Centrales de Córdoba o la región del Noroeste andino (TORRES, 1911b; SERRANO, 1931; LOPONTE, 2008). Debe agregarse que son escasas las referencias etnohistóricas que señalan el uso de adornos líticos entre las poblaciones locales, entre ellas figuran la de U. Schmidel quien menciona “...estos indios timbúes traen, en ambos lados de la nariz, embutida una estrellita de piedra blanca y azul" (SCHIMDEL, [1567] 1881: 27). También Rui Díaz de Guzmán señala que "...tienen la narices horadadas, donde sientan por gala en cada parte una piedra azul o verde..." (RUIDIAZ DE GUZMAN [1612]1882: 30-31). 
Con respecto a los moluscos marinos es muy posible que provengan de la costa atlántica bonaerense y/o de la República Oriental del Uruguay. En el caso de las cuentas y abalorios elaborados sobre $U$. haneti debieron tener una trayectoria más compleja dado que ejemplares con características similares han sido arqueológicamente identificados en la isla Martín García (Delta del Paraná) y en otros sitios localizados en las provincias de Santiago del Estero, Mendoza y Tucumán (OUTES, 1917; BOMAN, 1920; DOELLO JURADO, 1940; ACOSTA et al., 2015).

Finalmente, los metales fueron reconocidos tanto en sitios de grupos de cazadores-recolectores como en los atribuidos a la tradición tupiguaraní. La presencia de objetos metálicos ha sido señalada en distintas crónicas europeas del siglo XVI. Con relación a los grupos locales, FERNÁNDEZ DE OVIEDO Y VALDÉS (1944) menciona que "...los metales que tienen son cobre y latón o como latón; más esto lo traen de otras partes." (FERNÁNDEZ DE OVIEDO Y VALDÉS 1944, V: 131). Por su parte, Lope de Souza señaló que "... alguns delles furam os narises, e nos buracos trazem metidos pedaços de cobre mui lucentes..." (LOPE DE SOUSA, 1839: 54).

En cuanto a los guaraníes, el cronista Luis Ramírez expresa que, "...se llaman guarenis y por otro nombre chandris estos andan derramados por esta tierra y por otras muchas como cosarios a cavsa de ser enemigos de todas estotras naçiones y de otras muchas (...) estos señorean gran parte desta yndia y confinan con los que abitan en la sierra estos traen mucho metal de oro y plata en muchas planchas y orejeras y en achas con que cortan la montaña para senbrar (RAMÍREZ [1528] 2007: 31, el énfasis es nuestro).

En el registro arqueológico vimos que la frecuencia de objetos metálicos es muy baja y aislada. En cuanto a los hallados en contextos de cazadores-recolectores, los datos hasta ahora obtenidos sobre su composición mineralógica (básicamente cobre) y su diseño, sumado a la cronología de los sitios, indicarían que son de origen prehispánico y que provendrían de la región andina, muy posiblemente del Noroeste argentino (TORRES 1911B; LOPONTE, 2008; BONOMO et al., 2009). La presencia de algunos de ellos en contextos funerarios (TORRES 1911b) sugiere que debieron ser social y/o simbólicamente valorados. Cabe agregar que algunos datos (etnohistóricos y arqueológicos) indican que pudo existir una mayor disponibilidad de elementos metálicos dentro de los grupos guaraníes, siendo posible que los circuitos de abastecimiento hayan sido intraétnicos (ver discusión en LOPONTE \& ACOSTA, 2013).

En Arroyo Fredes la situación es más compleja ya que como señaláramos uno de los artefactos presenta un diseño atípico y una aleación que incluye cinc. La presencia de este elemento ha sido vinculada con la producción de ciertos artefactos elaborados luego de la conquista europea (e.g. CABANILLAS \& GONZÁLEZ, 2012). La cronología de este sitio, en donde también se recuperó una cuenta de vidrio veneciana (LOPONTE et al., 2011), sugiere el posible origen post-hispánico de la pieza. Sin em- bargo, si consideramos el bajo porcentaje de cinc detectado en la pieza de Arroyo Fredes en relación a los altos valores de los conjuntos europeos, no podemos descartar la utilización de una mena local de la región andina (ver BUC et al., 2013).

De cualquier manera, los tres tipos de materiales mencionados (rocas verdes, moluscos marinos y metales) debieron involucrar redes de intercambio en las que estuvieron incluidas poblaciones cultural y geográficamente distanciadas del HPI. La mayoría de estos artefactos pueden considerarse como objetos costosos, no por el grado de elaboración que presentan, sino por el complejo entramado de relaciones sociales que debió implicar su adquisición, desde su lugar de origen hasta el de su destino final. La información (etnohistórica y arqueológica) sugiere que en el HPI los principales vectores relacionados con la circulación e intercambio de estos y otros bienes incluye a distintos sectores de la llanura pampeana y los ejes potámicos de los ríos Uruguay y Paraná. A través de este último habrían ingresado productos del Noroeste argentino y del área Chaco-santiagueña (TORRES, 1911b; SERRANO, 1931; LOTHROP, 1932; LOPONTE, 2008). Para simplificar, el flujo de bienes que habría existido entre el $\mathrm{HPI}$ y las regiones mencionadas indica que la trayectoria de los objetos de origen exótico implicó redes indirectas de abastecimiento dentro de espacios amplios y a distancias grandes (LOPONTE, 2008).

\section{5.- CONCLUSIONES}

La generación de extensas redes de intercambio se encuentra estrechamente relacionada con el incremento de la población y con una mayor interacción entre diferentes grupos y personas (PRICE \& BROWN, 1985; HAYDEN \& GARGETT, 1990; GAMBLE, 1993, entre otros). En el HPI, el intercambio, tanto a escala local como extra regional, constituyó un mecanismo eficaz para las poblaciones humanas, ya que no solo habría permitido el abastecimiento de diferentes productos (ver síntesis en LOPONTE, 2008), sino que también se constituyó en un mecanismo de minimización de riesgo a través del intercambio de información y la posibilidad de cooperación intra e intergrupal. Esto último se vincula con la generación de redes de alianzas y/o de actos rituales, lo cual incluso, bajo ciertas condiciones, pudo favorecer o incrementar el flujo génico entre poblaciones (AMES, 1985; KIPNIS, 2002; TRUBBIT, 2003; WHALLON, 2006). En otras palabras, la mayor participación y conectividad de los individuos a través de extensas redes sociales puede concebirse como un "sistema de seguridad social" (sensu STINER, 2014). El mantenimiento de estos sistemas requiere, entre otros aspectos, del intercambio de dones, siendo los adornos un medio eficaz para este fin ya que por su reducido tamaño son fácilmente transportables y, además, al ser elaborados sobre materias primas perdurables, pueden ser transferidos de manera eficiente a través de grandes extensiones de tiempo y espacio (STINER, 2014). En el HPI esta situación se habría potenciado por el uso de canoas, 
tipo de adornos, en el marco de la relación establecida entre emisores-receptores, pudo no ser unívoca. Es decir que al tratarse de objetos susceptibles de ser conservados, su utilización pudo involucrar a varias generaciones de usuarios, por lo que es posible que el significado haya variado a través del tiempo. Por todo ello, si bien aquí hemos efectuado algunas conjeturas sobre la posible función simbólica de ciertos adornos, tales como su uso como amuletos o por su potencial vinculación con las actividades cinegéticas, arqueológicamente es muy difícil decodificar los "verdaderos" significados que pudieron tener para sus usuarios. Más allá de esto, los ornamentos constituyeron un segmento de la tecnología que ofreció ciertas ventajas a las poblaciones locales. En principio, habría permitido aumentar la cohesión social a nivel grupal y afrontar distintas necesidades sociales y económicas dentro de un ambiente que se tornó más competitivo producto del incremento demográfico y de la complejidad social que habría caracterizado a las poblaciones humanas que ocuparon el HPI durante la fase final del Holoceno tardío.

\section{6.- AGRADECIMIENTOS}

Queremos agradecer especialmente a Damián Voglino por la fotografía y la descripción técnica del molar modificado de $\mathrm{H}$. hydrochaeris proveniente del sitio Anahí. Las observaciones realizadas por el evaluador contribuyeron a mejorar la claridad del artículo. Este trabajo fue financiado por los subsidios PICT2011-2035, 2012-0215 y PIP2012-11220110100565. Cualquier error u omisión que contenga este trabajo es exclusiva responsabilidad de los autores.

\section{BIBLIOGRAFÍA} les pudieron ser materializados y expresados a través de la elaboración y del uso de adornos personales. Es posible que ciertos objetos hayan tenido un alto poder de señalización dentro del grupo de pertenencia, como sería el caso de los ornamentos locales. De igual modo pudo ocurrir con los de origen exótico, aunque estos últimos pudieron cobrar diferentes sentidos, ya que su adquisición implicó trayectorias más complejas, razón por la que también su uso pudo estar sujeto a distintas lecturas o significados. Se ha considerado que las señales costosas aumentan cuando los objetos son raros o exóticos; los cuales, además, suelen presentar una frecuencia baja en el registro. Al tratarse de artefactos especialmente valorados y conservado,s tienden a tener una tasa de pérdida baja, lo cual también incide en la escasa representación arqueológica (Quinn, 2006) que poseen la mayoría de estos ornamentos. La adquisición de elementos alóctonos, como los adornos elaborados sobre metal, rocas verdes y moluscos marinos, habría implicado la existencia de contactos interétnicos, a través de una extensa red de intercambio que debió incluir, como ya señalamos, grupos geográficamente distanciados.

Sin embargo, es necesario tener en cuenta que la información transmitida (social o simbólica) a través de este
ACOSTA, A

2005 Zooarqueología de cazadores-recolectores del extremo nororiental de la provincia de Buenos Aires (humedal del río Paraná inferior, Región pampeana, Argentina). Tesis Doctoral. Universidad Nacional de La Plata. La Plata.

ACOSTA, A., ALI, S., BUC, N., DAVRIEUX, N., DI PRIETO, V., LOPONTE, D., MAZZA, B., MUCCIOLO, L., PÉREZ, M., RIOS ROMAN, V., SACUR SILVESTRE, R., VIGLIOCCO, D., ZATTERA, R.

2013 Variabilidad arqueológica en el tramo final del humedal del Paraná inferior durante el Holoceno tardío. V Encuentro de discusión arqueológica del Noreste Argentino. Goya. 14 al 16 de Agosto.

ACOSTA, A., BUC, N., RAMíREZ, M., PREVOSTI, F., LOPONTE, D.

2014 Producción y uso de objetos ornamentales elaborados sobre dientes de carnívoros en contextos arqueológicos del humedal del Paraná inferior. Revista del Museo de Antropología. No publicado.

ACOSTA, A., LOPONTE, D.

2013 Complejidad social y estrategias de subsistencia de las poblaciones cazadoras-recolectoras del humedal del $\mathrm{Pa}$ raná inferior. Cuadernos (series especiales) 1(4), 60-74. 


\section{ACOSTA, A., LOPONTE, D., GARCÍA ESPONDA, C.}

2011 Primer registro de perro doméstico prehispánico (Canisfamiliaris) entre los grupos cazadores recolectores del humedal de Paraná inferior (Argentina). Antípoda. Revista de Antropología y Arqueología 13, 175-199.

\section{ACOSTA, A., MAZZA, B.}

2015 Restos óseos humanos y faunísticos: su relación en el espacio mortuorio en contextos de cazadores-recolectores del humedal del Paraná inferior (Argentina). Pesquisas, Antropología. No publicado.

\section{ACOSTA, A., PASTORINO, G. LOPONTE, D.}

2015 Registro de moluscos marinos entre grupos cazadores recolectores del sector centro-oriental de la región pampeana (Argentina). No publicado.

\section{ACOSTA, A., RIOS ROMÁN, V.}

2013 Explotación prehispánica de palmeras pro grupos cazadores-recolectores y horticultores del extremo sur de Sudamérica: el caso del huemdal del Paraná inferior (Argentina). Pesquisas Antropología 70, 197-216.

\section{AMBROSETTI, J.B.}

1895 Los cementerios prehistóricos del Alto Paraná (Misiones). Boletín del Instituto Geográfico Argentino 16, 227-263.

1905 El bronce en la región Calchaquí. Anales del Museo Nacional 11, 163-314.

AMES, K.M.

1985 Hierarchies, stress and logistical strategies among hunter-gatherers in northwestern North America, en PRICE, T.D. \& BROWN, J.A., Prehistoric Hunter-Gatherers: The Emergence of Cultural Complexity. 155-180. Academic Press. San Diego.

ARRIZURIETA, M.P., BUC, N., MAZZA, B., MUCCIOLO, L., MUSALI, J., PARISI, F., PAU, D., PERREZ, M. POGGGI, M., SILVESTRE, R.

2010 Nuevos aportes a la arqueología del sector continental del humedal del Paraná inferior, en Bárcena, J.R. \& Chiavazza, H., Arqueología Argentina en el Bicentenario de la Revolución de Mayo. Tomo V: 1793-1798. Universidad Nacional de Mendoza. Mendoza.

BAEZA, J.

2013 A propósito de algunas posibles relaciones con el NOA. Cuadernos del Instituto Nacional de Antropología y Pensamiento Latinoamericano - Series Especiales1 (4), 75-80-

BERNAL, V.

2008 Procesos de diferenciación biológica entre poblaciones humanas del Holoceno tardío de Patagonia. Una aproximación desde la variación métrica dental. Tesis doctoral. Universidad Nacional de La Plata. La Plata.

BETTINGER, R.L., BOYD, R., RICHERSON, P.J.

1996 Style, function, and cultural evolutionary processes, en H.D.G. Maschner, Darwinian Archaeologies. 133-164. Plenum. New York.

BLIEGE BIRD, R, SMITH, E.A.

2005 Signaling Theory, Strategic Interaction, and Symbolic Capital. Current Anthropology 46 (2), 221-248.
BLIEGE BIRD, R, SMITH, E.A., BIRD, D.W.

2001 The hunting handicap: Costly signaling in human foraging strategies. Behavioral Ecology and Sociobiology, 50: 9-19.

BOMAN, E.

1920 Cementerio indígena en Viluco (Mendoza) posterior a la conquista. Anales del Museo Nacional de Historia Natural de Buenos Aires XXX, 501-559.

BONOMO, M.

2013 Reanálisis de la colección de Samuel Lothrop procedente del delta del Paraná. Relaciones de la Sociedad Argentina de Antropología 38 (1), 169-198.

\section{BONOMO, M., CAPDEPONT, I., MATARRESE A.}

2009 Alcances en el estudio de colecciones. Los materiales arqueológicos del Delta del río Paraná depositados en el museo de La Plata (Argentina). Arqueología Suramericana 5 (1), 68-101.

BUC, N.

2012 Tecnología Ósea de Cazadores-Recolectoresdel Humedal del Paraná inferior. Serie Monográfica II, Arqueología de la Cuenca del Plata, Instituto Nacional de Antropología yPensamiento Latinoamericano. Buenos Aires.

BUC, N., CABANILLAS, E., DAVRIEUX, M.N.

2014 Objetos metálicos entre grupos Guaraníes del Delta del Paraná. Holoceno tardío, Buenos Aires - Argentina. Revista do Museu de Arqueologia e Etnologia 24. No publicado.

BUC, N., CAGGIANO, M.A.

2015 Revisando colecciones de la cuenca inferior del Plata. Cuadernos (series especiales). Instituto Nacional de Antropología y Pensamiento Latinoamericano. En prensa.

\section{BURKART, R., BÁRBARO, N., SÁNCHEZ, R., GÓMEZ, D.}

1999 Ecoregiones de la Argentina. Administración de Parques Nacionales, Programa de Desarrollo Institucional Ambiental, Secretaría de Recursos Naturales y Desarrollo Sustentable. Buenos Aires.

\section{CABANILLAS, E.D., GONZÁLEZ, L.R.}

2012 Aleaciones y estilo en épocas de contacto hispano-indígena en el noroeste argentino. Revista de Arqueología Histórica Argentina y Latinoamericana 6, 185-198.

\section{CAGGIANO, M.A.}

1984 Prehistoria del NE Argentino y sus vinculaciones con la República Oriental del Uruguay y Sur de Brasil. Pesquisas, Antropología 38, 5-109.

\section{CAVALLOTTO, J.L., VIOLANTE, R. PARKER, G.}

1999 Historia evolutiva del Río de la Plata durante el Holoceno. Actas del XIV Congreso Geológico Argentino I, 508-515.

2004 Sea-level fluctuations during the last $8600 \mathrm{yr}$ in the de la Plata River (Argentina). Quaternary International 114, 155-165. 
HILDEBRANDT, W.R., MCGUIRE K.R.

2003 Large-Game Hunting, Gender-Differentiated Work Organization, and the Role of Evolutionary Ecology in California and Great Basin Prehistory: A Reply to Broughton and Bayham. American Antiquity 68, 790-792.

HILL, E.

2013 Archaeology and Animal Persons Toward a Prehistory of Human-Animal Relations Environment and Society: Advances in Research 4 (1), 117-136.

HILL, K., HURTADO, A.M.

1996 Ache life history: the ecology and demography of a foraging people. Hawthorne. New York.

KIPNIS, R.

2002 Long term land tenure systems in Central Brazil: evolutionary ecology, risk management, and social geography, en FITZHUGH, B. \& HABU, J. Beyond Foraging and Collecting, Evolutionary Change in Hunter-Gatherer Settlement Systems. 181-231. Kluwer Academic- Plenum Publishers. New York.

KUHN, S.L.

2014 Signaling theory and technologies of communication in the paleolithic. Biological Theory 9, 42-50.

FITZHUGH, B.S., PHILLIPS, C., GJESFJELD, E.

2011 Modeling Variability in Hunter-Gatherer Information Networks: An Archaeological Case Study from the Kuril Islands, en WHALLON, R., LOVIS, W., R. HITCHCOCK, R. Information and its Role in Hunter-Gatherer Bands. 85115. Cotson Institute for Archaeology. Los Angeles.

GAMBLE, C.

1993 Exchange, Foraging and Local Hominid Networks, en SCARRE, C.\& HEALY, F., Trade and Exchange in Prehistoric Europe. 35-44. Oxbow Books. Oxford.

GLUZMAN, G.A., GONZÁLEZ, L.

2009 El contacto hispano-indígena a través de la metalurgia del antiguo noroeste de Argentina. Actas VII Congreso Ibérico de Arqueometría, 522-530.

GRAMAJO DE MARTÍNEZ MORENO, A.J.

1979. El Contacto Hispano Indígena en Santiago del Estero con especial referencia a la cerámica. Serie Estudio 2:3-67.

HAYDEN, B., GARGETT, R.

1990 Big man, big heart? A Mesoamerican view of the emergence of complex societies. Ancient Mesoamerica 1 (1), 3-20.

HAWKES, K, O'CONNELL, J., BLURTON JONES, NB,

1997 Hadza women's timeallocation, offspring provisioning, and the evolution of long postmenopausal life spans. Current Anthropology 38, 551-577.

HEGMON, M.

1992 Archaeological Research on Style. Annual Review of Anthropology 21, 517-536.

HENSHILWOOD, C.S., D'ERRICO, F., VANHAEREN, M., VAN NIEKERK, K., JACOBS, Z.

2004 Middle Stone Age shell beads from South Africa. Science 304, 404.
KUHN S.L., STINER, M.C.

2007a Body ornamentation as information technology: towards an understanding of the significance of early beads, en $P$. MELLARS, K. BOYLE, O. BAR-YOSEF, C. STRINGER, Rethinking the human revolution. 45-54. McDonald Institute monographs, University of Cambridge Press. Cambridge.

2007b Palaeolithic ornaments: implications for cognition, demography and identity. Diogenes 214:40-48

\section{KMUSSER}

2010 http://creativecommons.org/licenses/by-sa/3.0)], Wikimedia Commons. https://commons.wikimedia.org/wiki/File\% 3ARiodelaplatabasinmap.png.

\section{LOPE DE SOUZA, P.}

1839 Diario da Navegação da armada que foi á terra do Brasil em 1530 sob a Capitania-Mor de Martin Affonso de Sousa. Typographia da Sociedade propagadora dos conhecimentos uteis Lisboa. Lisboa.

LOPONTE, D.

2008 Arqueología del Humedal del Paraná Inferior. Arqueología de la Cuenca del Plata, Serie Monográfica 1. Ediciones del Riel. Buenos Aires.

LOPONTE, D., ACOSTA, A.

2007 Horticultores amazónicos en humedal del Paraná inferior, los primeros datos isotópicos de la dieta. En: Arqueología en las Pampas. C. Bayón, A. Pupio, M. I. González, N. Flegenheimer, M. Frére (Eds.): Tomo I, 75-93. Sociedad Argentina de Antropología, Buenos Aires. extremo meridional de su distribución geográfica. Cuadernos Instituto Nacional de Antropología y Pensamiento Latinoamericano - Series Especiales 1(4), 193-235.
2013 La construcción de la unidad arqueológica Guaraní en el 
LOPONTE D., ACOSTA, A., CAPPARELLI, I., PÉREZ, M.

2011 La arqueología guaraní en el extremo meridional de la cuenca del Plata, en ACOSTA, A. \& LOPONTE, D., Arqueología Tupíguaraní. 111-154. Instituto Nacional de Antropología y Pensamiento Latinoamericano. Buenos Aires.

LOPONTE, D., ACOSTA, A., MUCCIOLO, L.

2012 Contribución a la arqueología del delta del Paraná: El nivel acerámico del sitio Isla Lechiguanas 1. Comechingonia 16 (1), 229-268.

LOPONTE, D., ACOSTA, A., MUSALI, J.

2006 Complexity among Hunter-Gatherers from the Pampean Region South America, en GRIER, C., JANGSUK, K., UCHIYAMA, J., Beyond Affluent Foragers. Rethinking Hunter-Gatherer Complexity. 106-125. Oxbow Books. Oxford.

\section{LOPONTE, D., CORRIALE, M.J.}

2013 Isotopic values of diet of Blastocerus dichotomus (marsh deer) in Paraná Basin, South America. Journal of Archaeological Science 40, 1382-1388.

\section{LOTHROP, S.}

1932 Indians of the Paraná Delta River. Annals of the New York Academy of Sciences XXXII, 77-232. New York.

\section{MAYNARD SMITH, J., HARPER, D.G.C.}

2003 Animal signals. Oxford University Press. Oxford.

\section{MAZZA, B., D. LOPONTE}

2012 Las prácticas mortuorias en el humedal del Paraná inferior. Arqueología Iberoamericana 13, 3-21.

MENGHIN, O.F.A.

1957 El Poblamiento Prehistórico de Misiones. Anales de Arqueología y Etnología XII, 19-40.

MITSCH, W.J., GOSSELINK, J.G.

1986 Wetlands. Van Nostrand Reinhold. New York.

\section{MÜLLER, F.}

1989 [1935] Etnografía de los Guaraní del Alto Paraná. Socieatis Verbi Divini. Rosario.

MUSCIO, $\mathrm{H}$.

2002 Señales costosas y comportamientos rituales en sociedades de cazadores recolectores de los Andes Centro-Sur: la hipótesis de display. Cuaderno Di-Thule XXIII Convegno Internationzionale di Americanistas. Perugia: 1-12.

NEIFF, J.J.

1999 El régimen de pulsos en ríos y grandes humedales de Sudamérica, en MALVÁREZ A., Tópicos sobre humedales subtropicales y templados de Sudamérica. 97-146. Universidad de Buenos Aires. Buenos Aires.

OUTES, F.

1917 Primer hallazgo arqueológico en la Isla Martín García. Anales de la Sociedad Científica Argentina XXXII, 265-277.

1918 Nuevos rastros de la cultura Guaraní en la cuenca del Paraná inferior. Anales de la Sociedad Científica Argentina LXXXII, 153-182.
PÉREZ, M.

2010 Tecnología de producción de la alfarería durante el Holoceno tardío en el humedal del Paraná inferior. Un estudio petrográfico. Tesis de Licenciatura. Universidad de Buenos Aires. Buenos Aires.

PÉREZ, M., CAÑARDO, L.

2004 Producción y uso de cerámica en el norte de la provincia de Buenos Aires, en MARTíNEZ, G., GUTIERREZ, M., CURTONI, R., BERÓN, M., MADRID, P., Aproximaciones contemporáneas a la arqueología pampeana. Perspectivas teóricas, metodológicas, analíticas y casos de estudio. 335349. UNCPBA, Facultad de Ciencias Sociales. Olavarría.

\section{PETROCELLI, J.}

1975 Nota preliminar sobre hallazgos arqueológicos en el Valle del Río Luján (Población Río Luján, Campana, Provincia de Buenos Aires). Actas I Congreso Arqueología Argentina. Rosario: 251-270.

PRICE, T.D., BROWN, J.A.

1985 Aspects of hunter-gatherer complexity, en PRICE, T.D. \& BROWN, J.A., Prehistoric Hunter-Gatherers: The Emergence of Cultural Complexity. 3-20. Academic Press. Orlando.

PROUS, A.

2011 Estudios sobre los portadores de la cerámica tupiguarani en Brasil: proto-Tupi, proto-Guarani y otros.... en LOPONTE, D.M., ACOSTA, A., Arqueología Tupiguaraní. 23110. Instituto Nacional de Antropología y Pensamiento Latinoamericano. Buenos Aires.

QUINN, C.P.

2006 Vital signs: costly signalng and personal adornment in the near Eastern early Neolithic. Master Thesis. Washington State University. Washington.

2015 Signals in stone: exploring the role of social information exchange, conspicuous consumption, and costly signaling theory in lithic analysis, en GOODALE, N., ANDREFSKY, W., Lithic Technological Systems and Evolutionary Theory. 198-222. Cambridge University Press. Cambridge.

\section{RAMÍREZ, L.}

[1528] 2007. Carta de Luis Ramírez a su padre desde el Brasil (1528): Origenes de lo 'real maravilloso' en el Cono Sur. Edición, Introducción y notas de Juan Francisco Maura. Colección Textos de la revista Lemir. 2007. Edición electrónica: http://parnaseo.uv.es/Lemir/Textos/Ramirez.pdf

\section{REICHLEN, $\mathrm{H}$.}

1940 Recherches archéologiques dans la Province de Santiago del Estero (Rép. Argentine). Journal de la Société des Américanistes 32 (1), 133 - 237.

ROSSANO, M.J.

2010 Making Friends, Making Tools, and Making Symbols. Current Anthropology 51 (S1), S39-S54.

\section{RUIDIAZ DE GUZMAN}

[1612] 1882. Argentina, Historia del Descubrimiento, Conquista y Población del Río de la Plata. Casavalle Editor. Buenos Aires. 
SACUR SILVESTRE, R., BUC, N., ACOSTA, A., LOPONTE, D.

2013 Estrategias de captura de presas y sistemas de armas de los cazadores-recolectores que habitaron el humedal del Paraná inferior: una aproximación experimental y arqueológica. Comechingonia 17, 27-57.

\section{SALEMME, M.}

1987 Paleoetnozoología del Sector Bonaerense de la Región Pampeana. Tesis doctoral. Universidad Nacional de La Plata. La Plata.

SCHIFFER, M.B.

1976 Behavioral archaeology. Academic Press. New York.

SCHMIDEL, U.

[1567] 1881. Historia y descubrimiento del Río de la Plata y Paraguay. Imprenta y Librería de Mayo. Buenos Aires.

\section{SERRANO, A.}

1931 Arqueologia del litoral. Memorias del Museo de Paraná IV. Paraná.

1972 Líneas fundamentales de la arqueología del litoral (una tentativa de periodización). Instituto de Antropología, Universidad Nacional de Córdoba. Córdoba.

SMITH, E.A., BLIEGE BIRD, R., BIRD D.W.

2003 The benefits of costly signaling: Meriam turtle hunters. Behavioral Ecology 14 (1), 116-126

SOSIS, R., ALCORTA, C.

2003 Signaling, solidarity, and the sacred: the evolution of religious behavior. Evolutionary Anthropology 12, 264-274.

STERELNY, K.

2014 A paleolithic reciprocation crisis: symbols, signals, and norms. Biological Theory 9, 65-77.

STINER, M. C.

2014 Finding a common bandwidth: causes of convergence and diversity in paleolithic beads. Biological Theory 9, 51-64.

STINER, M.C., KUHN, S., GÜLEK, E.

2013 Early Upper Paleolithic shell beads at Üça-gızlı Cave I (Turkey): Technology and the socioeconomic context of ornament life-histories. Journal of Human Evolution 64, 380-398.

\section{TABORIN, Y.}

1993 La Parure en Coquillage au Paléolithique. Gallia Préhistoire29.

TAPIA, A.H., LANDA, C., DE ROSA, H., MONTANARI, E.

2009 Artefactos metálicos de las inhumaciones del "Cementerio Indígena" de Baradero, en Arqueometría Latinoamericana, 263-293. Comisión Nacional de Energía Atómica. Buenos Aires.

TONELLO, M.S., PRIETO, A.

2010 Tendencias climáticas para los pastizales pampeanos durante el Pleistoceno tar $\neg$ dío Holoceno: estimaciones cuantitativas basadas en secuencias polínicas fósiles. Ameghiniana 47 (4), 501-514.
TORRES, L.M

1911a El totemismo. Su origen, significado, efectos y supervivencias. Anales del Museo Nacional de Buenos Aires 20, 485-553.

1911b Los Primitivos Habitantes del Delta del Paraná. Universidad Nacional de La Plata, Biblioteca Centenaria. La Plata.

TRUBBIT, M.B.

2003 The Production and Exchange of Marine Shell Prestige Goods. Journal of Archaeological Research 11 (3): 243-277.

VANHAEREN, M., D'ERRICO, F., VAN NIEKERK, K., HENSHILWOOD, C.S., ERASMUS, R.M.'

2013 Thinking strings: additional evidence for personal ornament use in the Middle Stone Age of Blombos Cave South Africa. Journal of Human Evolution 64, 500-517.

VIOLANTE, R., PARKER, G.

2004 The post-glacial maximum trangression in the de la Plata River and adjacent continental shelf, Argentina. Quaternary International 114, 167-181.

WHALLON, R.

2006 Social networks and information: non- "utilitarian" mobility among huntergatherers. Journal of Anthropological Archaeology 25, 259-270.

WHITE, R.

1999 Integrating social and operational complexity: the material construction of social identity at Sungir en AVERBOUH A., CATTELAIN, P., JULLIEN, M., L'Os: Festschrift for Henriette Camps-Fabrer. 120-37. Université de Marseille. Aixen-Provence.

2007 Systems of Personal Ornamentation in the Early Upper Palaeolithic: Methodological Challenges and New Observations, en MELLARS, P., BOYLE, K., BAR-YOSEF, O., STRINGER C Rethinking the Human Revolution: New Behavioral and Biological Perspectives on the Origin and Dispersal of Modern Humans. 287-302. McDonald Institute for Archaeological Research Cambridge. Cambridge.

WIESSNER, P.

1983 Style and Social Information in Kalahary San Projectile Points, American Antiquity 48 (2), 253-276.

1984 Reconsidering the behavioral basis for style: a case study among the Kalahari San. Journal Anthropology Archaeology 3, 190-234

2002 Hunting, Healing, and HxaroExchange: A Long-Term Perspective on !Kung (Ju/'hoansi) Large-Game Hunting. Evolution and Human Behavior 23, 407-436.

WOBST, H. M.

1977 Stylistic Behavior and Information Exchange, en CLELAND, C.E. For the Director: Research Essays in Honor of James B. Griffin. 317-342. University of Michigan Anthropology Papers 61 . Ann Arbor.

ZAHAVI, A.

1975 Mate Selection: A Selection for Handicap. Journal of Theoretical Biology 53 (1), 205-214.

ZUCOL, A.F., LOPONTE, D.M.

2005 Phytolith fertility study and methodological comparative analysis in human teeth tartar of archaeological sites of Buenos Aires province (Argentina). The Phytolitarien 17(2), 15 
